This document is confidential and is proprietary to the American Chemical Society and its authors. Do not copy or disclose without written permission. If you have received this item in error, notify the sender and delete all copies.

\title{
Matrix Isolation Spectroscopy and Nuclear Spin Conversion of Propyne Suspended in Solid Parahydrogen
}

\begin{tabular}{|r|l|}
\hline Journal: & The Journal of Physical Chemistry \\
\hline Manuscript ID & Draft \\
\hline Manuscript Type: & Article \\
\hline Date Submitted by the & $\mathrm{n} / \mathrm{a}$ \\
\hline Complete List of Authors: & $\begin{array}{l}\text { Strom, Aaron; University of Wyoming, Chemistry } \\
\text { Gutierrez-Quintanilla, Alejandro; Aix-Marseille Université, Laboratoire } \\
\text { PIIM, Team ASTRO } \\
\text { Chevalier, Michele; Universite Paris-Saclay } \\
\text { Ceponkus, Justinas; Vilniaus Universitetas, Institute of Chemical Physics } \\
\text { Crepin, Claudine; Institut des Sciences Moleculaires d'Orsay, } \\
\text { Anderson, David; University of Wyoming, Department of Chemistry }\end{array}$ \\
\hline
\end{tabular}

\section{SCHOLARONE ${ }^{\text {m }}$ Manuscripts}




\title{
Matrix Isolation Spectroscopy and Nuclear Spin Conversion of Propyne Suspended in Solid Parahydrogen
}

\author{
A. I. Strom, ${ }^{1}$ A. Gutiérrez-Quintanilla, ${ }^{2,3 a}$ M. Chevalier, ${ }^{2}$ J. Ceponkus, ${ }^{4}$ C. Crépin, ${ }^{2}$ and D. T. \\ Anderson $^{1 b}$ \\ ${ }^{1}$ Department of Chemistry, University of Wyoming, Laramie, WY 82071-3838, USA \\ ${ }^{2}$ Université Paris-Saclay, CNRS, Institut des Sciences Moléculaires d'Orsay, 91405, \\ Orsay, France \\ ${ }^{3}$ Instituto Superior de Tecnologías y Ciencias Aplicadas (InSTEC), Universidad de La Habana, \\ Ave. Salvador Allende No. 1110, Quinta de los Molinos, La Habana 10400, Cuba \\ ${ }^{4}$ Institute of Chemical Physics, Vilnius University, Sauletekio ave. 9 III, LT-10222 Vilnius, \\ Lithuania \\ aPresent address: Aix-Marseille Université, Laboratoire PIIM, Team ASTRO, Service 252, Saint \\ Jérôme, Ave. Escadrille Normandie Niemen, 13013 Marseille, France. \\ b)Author to whom correspondence should be addressed: danderso@uwyo.edu
}

\begin{abstract}
Parahydrogen $\left(\mathrm{pH}_{2}\right)$ quantum solids are excellent matrix isolation hosts for studying the rovibrational dynamics and nuclear spin conversion (NSC) kinetics of molecules containing indistinguishable nuclei with nonzero spin. The relatively slow NSC kinetics of propyne $\left(\mathrm{CH}_{3} \mathrm{CCH}\right)$ isolated in solid $\mathrm{pH}_{2}$ is employed as a tool to assign the rovibrational spectrum of propyne in the $600-7000 \mathrm{~cm}^{-1}$ region. Detailed analyses of a variety of parallel $(\Delta K=0)$ and perpendicular $(\Delta K=1)$ bands of propyne indicate that the end-over-end rotation of propyne is quenched, but $K$ rotation of the methyl group around the $C_{3}$ symmetry axis still persists. However, this single-axis $K$ rotation is significantly hindered for propyne trapped in solid $\mathrm{pH}_{2}$ such that the energies of the $K$ rotational states do not obey simple energy level expressions. The NSC kinetics of propyne follows first-order reversible kinetics with a 287(7) min effective time constant at $1.7 \mathrm{~K}$. Intensity-intensity correlation plots are used to determine the relative line strengths of individual ortho- and para-propyne rovibrational transitions, enabling an independent estimation of the ground vibrational state effective $A^{\prime \prime}$ constant of propyne.
\end{abstract}




\section{Introduction}

Spectroscopy of dopant molecules isolated in quantum condensed matter affords unique opportunities to probe the properties of weakly interacting, light mass media at the molecular level. In particular, inherently large amplitude dopant rotational motion is an incredibly useful probe of the quantum host dynamics determined by both kinetic and potential energy contributions, as one of the main qualifications of quantum condensed matter is the significance of the zero-point energy relative to the strength of the intermolecular forces. ${ }^{1-4}$ Rovibrational spectroscopy of molecules and clusters embedded in ${ }^{4} \mathrm{He}$ nanodroplets has long been used to probe the microscopic dynamics of this unique quantum fluid, where rotational fine structure is usually observed due to the Bose-Einstein statistics associated with ${ }^{4} \mathrm{He} .{ }^{5-9}$ The rovibrational spectra of dopants dissolved in liquid He nanodroplets are representative of the gas-phase molecular or cluster symmetry and yet the rotational constants are reduced compared to the isolated molecule. Solid parahydrogen $\left(\mathrm{pH}_{2}\right)$ provides another opportunity to study dopant rotation. However, stronger $\mathrm{H}_{2}-\mathrm{H}_{2}$ intermolecular forces relative to the intermolecular forces in He and the hexagonal close packed crystal structure in the solid $\mathrm{pH}_{2}$ limit the propensity for observing dopant rotation in $\mathrm{pH}_{2}$ matrices. Molecules that occupy single substitutional sites in the $\mathrm{pH}_{2}$ crystal lattice $\left(\mathrm{CH}_{4},{ }^{10-13} \mathrm{NH}_{3},{ }^{14} \mathrm{H}_{2} \mathrm{O},{ }^{15,16} \mathrm{HCl},{ }^{17}\right.$ and $\mathrm{CO}^{18}$ to name published examples) undergo nearly free rotation because the potential barriers to rotation are extremely small in this highly symmetric solvation environment. When bigger molecules are confined in solid $\mathrm{pH}_{2}$ that occupy larger substitutional sites (double and larger), the end-over-end tumbling motions of the dopant are typically quenched. ${ }^{19,20}$ Yet molecules containing methyl rotors $\left(\mathrm{CH}_{3}\right)$ that behave as symmetric tops, such as $\mathrm{CH}_{3} \mathrm{~F},{ }^{21}$ or internal rotors, like $\mathrm{CH}_{3} \mathrm{OH},{ }^{22}$ can continue to show spinning 
motion of the $\mathrm{CH}_{3}$ rotor. This spinning motion can be used to probe quantum solvation in larger, more complex molecules, such as acetylacetone $\left(\mathrm{CH}_{3}(\mathrm{C}=\mathrm{O}) \mathrm{CH}_{2}(\mathrm{C}=\mathrm{O}) \mathrm{CH}_{3}\right) \cdot{ }^{23,24}$

The accessible temperature range is an important aspect of probing the rotational motions of dopants trapped in quantum condensed matter. All of the molecules that demonstrate rotation while solvated in solid $\mathrm{pH}_{2}$ have small moments of inertia ( $\mathrm{CO}$ being the only exception in the literature ${ }^{18}$ ), and, thus, the rotational motion endures in spite of the rotational barriers induced by the $\mathrm{pH}_{2}$ host. This means that the energy of the lowest excited rotational state of the dopant is also large with respect to the available thermal energy in the accessible temperature range in solid $\mathrm{pH}_{2}(1.5$ to $4.3 \mathrm{~K})$, so there is very little population in excited rotational levels that significantly limits the number of rovibrational transitions that can be observed. Nonetheless, closer examination of the list of rotating molecules reveals another important attribute, namely indistguishable hydrogen atoms. As a consequence of the symmetrization postulate of quantum mechanics, ${ }^{25}$ excited rotational states connected to the different nuclear spin isomers of the dopant species can become kinetically trapped. Optimally, one would like to observe multiple rovibrational transitions to help tease apart different rotational and vibrational contributions to the measured transition energies in order to maximize the information content on the rotational dynamics of the dopant isolated in solid $\mathrm{pH}_{2}$. Of course, nuclear spin constraints are active for methyl rotors in symmetric top molecules with two different moments of inertia, such as $\mathrm{CH}_{3} \mathrm{~F}$. In this case, sufficient population of excited $K=1$ rotational states are kinetically trapped, such that transitions out of these levels are observable in the IR spectra of freshly deposited samples of $\mathrm{CH}_{3} \mathrm{~F}$ isolated in solid $\mathrm{pH}_{2}{ }^{21}$ With time, these transitions out of excited rotational states decrease in intensity due to condensed phase NSC. Thus, nuclear spin restrictions on the rotational wavefunctions provide the means to study dopant rotational motion in low temperature 
quantum hosts not possible in the fully equilibrated samples. This was successfully demonstrated for $\mathrm{CH}_{3} \mathrm{~F}^{21}$ and $\mathrm{CH}_{3} \mathrm{OH}^{22}$ doped $\mathrm{pH}_{2}$ solids.

Our groups, in Wyoming and Orsay, became interested in investigating the infrared spectroscopy and solid state dynamics of methyl rotors trapped in solid $\mathrm{pH}_{2}$ based on previous experiments conducted in Orsay. ${ }^{23,24}$ Preliminary results on the infrared spectroscopy of propyne in solid $\mathrm{pH}_{2}$, that are presented in the thesis of Gutiérrez, ${ }^{26}$ were provided to the Wyoming group to stimulate further collaborative studies. The idea is to develop useful spectroscopic tools to gain information about how the quantum cavity around the methyl rotor perturbs the large amplitude $K$ rotational motion. Specifically, we want to develop analytical methods that employ NSC phenomena to rigorously assign the measured infrared spectra for a number of molecular dopants containing methyl groups. For example, which types of vibrational modes provide the richest sources of information? If spectra contain significant fine structure, how can NSC be used to assign the different peaks? A number of researchers have already addressed some of these issues with much success, ${ }^{14,21-24,27}$ and we intend to refine the existing spectroscopic tools to better determine how solvation in quantum condensed matter perturbs rotational dynamics in dopant molecules. Furthermore, rovibrational spectroscopy can be used for NSC kinetics studies of methyl rotor molecules with different attributes. Ultimately, our goal is to measure signatures of NSC and quantitative nuclear spin populations of molecules for applications to photochemical and reactive studies conducted in solid $\mathrm{pH}_{2}$.

\section{METHODS}

Most of the spectroscopic data presented here was acquired in the laboratory in Wyoming. Some additional experiments were conducted in Orsay as this laboratory has the capability of performing prolonged studies of a single $\mathrm{pH}_{2}$ sample. A detailed description of the experimental 
methods used in Orsay is published elsewhere, ${ }^{28}$ and here we point out some important differences between the two laboratories. In the laboratory in Orsay, samples are not obtained by co-deposition but rather are deposited through a single deposition tube. The samples are thinner and the concentration of dopant is higher than the samples grown in Wyoming. The lowest temperature achieved in the laboratory in Orsay is $2.8 \mathrm{~K}$, the highest resolution of the FTIR spectrometer is $0.125 \mathrm{~cm}^{-1}$, and samples can be kept frozen for several days at a time. As such, the spectroscopic data from Orsay was recorded at 2.8 and $4.0 \mathrm{~K}$, for samples that were kept frozen for longer times and annealed, and thus could be used to complement and confirm the results from the Wyoming laboratory.

The experimental apparatus for $\mathrm{pH}_{2}$ matrix isolation spectroscopy in the Wyoming laboratory has been discussed previously, ${ }^{14,29}$ so only details relevant to this study are presented below. Propyne-doped $\mathrm{pH}_{2}$ solids are grown via the rapid-vapor-deposition (RVD) technique developed by Fajardo and Tam. ${ }^{30,31}$ This procedure involves the codeposition of independent $\mathrm{pH}_{2}$ and propyne gas streams onto a precooled $\mathrm{BaF}_{2}$ optical substrate held near $\sim 2.5 \mathrm{~K}$ within a sample-in-vacuum $l \mathrm{He}$ cryostat, producing millimeters-thick propyne-doped $\mathrm{pH}_{2}$-enriched samples in about $\sim 30$ mins. Enriched $\mathrm{pH}_{2}$ gas $(>99.97 \%)$ was rapidly introduced into the cryostat via the outlet tube of a variable-temperature ortho-to-para $(o / p)$ converter packed with granular $\mathrm{Fe}(\mathrm{OH})_{3}$ catalyst and backed by $800-1000$ torr of normal $\mathrm{H}_{2}$ gas (Linde, $\geq 99.999 \%$ ) passed through a needle-valve set to achieve flowrates of $\Phi_{H_{2}}=200-500 \mathrm{mmol} \cdot \mathrm{hr}^{-1}$. In this work, the o/p converter is operated around $15 \mathrm{~K}$ producing $\mathrm{pH}_{2}$ solids containing orthohydrogen $\left(\mathrm{oH}_{2}\right)$ concentrations $\approx 100 \mathrm{ppm}$ (approximated via gas-phase $\mathrm{H}_{2}$ rotational partition functions or determined experimentally using the $\mathrm{oH}_{2}$-induced $Q_{1}(0)$ feature ${ }^{31}$ and sample thickness ${ }^{32}$ ). Room temperature propyne (Aldrich, $\geq 99 \%$ as-received) was introduced into the cryostat 
through a separate needle-valve set to achieve acceptable working concentrations that favor isolated monomers and disfavor propyne clusters. Exclusively as-deposited samples (hexagonal close packed (hcp) fractions between $\approx 50-80 \%$, estimated using the $U_{1}(0)$ feature ${ }^{29}$ ) were studied in this work; annealing was conducted post-NSC to check for reversible and irreversible changes in measured IR spectra to confirm rovibrational assignments. Sample temperatures were measured using a pair of Si-diodes (attached to the OFHC $\mathrm{Cu}$ optical mount making thermal contact with the $\mathrm{BaF}_{2}$ window) connected to a digital temperature controller. One diode $\left(T_{\mathrm{A}}\right)$ is located near to the cold finger of the cryostat, with the second $\left(T_{\mathrm{B}}\right)$ at the end of the optical holder; $T_{\mathrm{A}}$ is typically too cold to register temperatures $(<1.0 \mathrm{~K})$, so sample temperatures are reported using $T_{\mathrm{B}} \geq 1.5 \mathrm{~K}$. Temperatures are recorded autonomously (sampling rate of $1 \mathrm{~Hz}$ ) using free chart recording software installed on the same computer used for spectroscopic measurements.

Total propyne monomer concentrations are measured via Beer's law, stated as ${ }^{29}$

$$
\left[\mathrm{CH}_{3} \mathrm{CCH}\right](\mathrm{ppm})=\frac{\ln (10) \int A_{i}(\tilde{v}) d \tilde{v}}{\varepsilon_{i} H_{2} d} V_{0}\left(1 \times 10^{6}\right)
$$

where $A_{i}(\tilde{v})=\log _{10}\left[I_{0}(\tilde{v}) / I(\tilde{v})\right]$ is the decadic absorbance of the $i^{t h}$ vibrational mode of propyne integrated over the entire band area $(\tilde{v}$ to $\tilde{v}+d \tilde{v}), \varepsilon_{i}^{p H_{2}}=1.06 \varepsilon_{l}^{\text {gas }}$ is the gas-phase integrated absorption coefficient corrected for the refractive index of solid $\mathrm{pH}_{2},{ }^{32} d$ is the thickness of the solid $p \mathrm{H}_{2}$ slab, ${ }^{32}$ and $V_{0}=23.16(6) \mathrm{cm}^{3} \mathrm{~mol}^{-1}$ is the molar volume of solid $p \mathrm{H}_{2}$ at $l$ He temperatures. ${ }^{33}$ Concentrations and $\mathrm{pH}_{2}$ samples thicknesses are reported as weighted averages weighted by uncertainties. Integrated intensities from the literature and integration protocols are provided in the Supporting Information, Table S2. Similarly, $\mathrm{pH}_{2}$ sample thicknesses are reported as the weighted average of three independent measurements, integrated intensities of the $Q_{1}(0)+S_{0}(0), S_{1}(0)+S_{0}(0)$ double transitions and $Q_{R}(0)$ IR absorptions bands ${ }^{32}$ 
weighted by their absorption coefficients. Error propagation is performed in the usual way, in which experimental as well as reported uncertainties in the literature are considered; typically, the largest contribution to the stated errors stem from uncertainties in the integrated absorption coefficients. A high-resolution FTIR spectrometer (Bruker IFS 120/5; $\Delta \tilde{v}=0.02-0.03 \mathrm{~cm}^{-1}$ ) optimized for normal incidence transmission optical measurements was employed using multiple optical setups: 1) a globar (GB; MIR) source, Ge coated $\mathrm{KBr}$ beamsplitter and $l \mathrm{~N}_{2}$ cooled InSb detector, 2) a GB source, Ge coated $\mathrm{KBr}$ beamsplitter and $l \mathrm{~N}_{2}$ cooled $\mathrm{HgCdTe}$ (MCT) detector, 3) a tungsten filament (W; NIR) with $\mathrm{a} \mathrm{CaF}_{2}$ beamsplitter and 4) identical to setup \#2 with the addition of a long pass filter (LPF) with a $3861 \mathrm{~cm}^{-1}$ cutoff in the IR beam path to eliminate absorptions by the $p \mathrm{H}_{2}$ host. These optical setups were designed to record highfidelity IR spectra of specific vibrational modes of propyne located within the allowed wavenumber ranges $\left(\Delta \tilde{v}=0.03 \mathrm{~cm}^{-1}\right.$ over $600-4800 \mathrm{~cm}^{-1}$ for the MCT and $\Delta \tilde{v}=0.02 \mathrm{~cm}^{-1}$ over $1800-5000 \mathrm{~cm}^{-1}$ or $1800-10,000 \mathrm{~cm}^{-1}$ for $\mathrm{InSb}$ with the Ge coated $\mathrm{KBr}$ or $\mathrm{CaF}_{2}$ beamsplitters, respectively). Furthermore, propyne concentrations deliberately span two orders of magnitude (roughly 20-300 ppm) in order to enhance the sensitivity of target mode(s) in each experiment.

In order to study the NSC of propyne molecules isolated in solid $\mathrm{pH}_{2}$ below $l \mathrm{He}$ temperatures, room temperature populations of excited rotational states of propyne must be deposited into the solid much faster than they can relax, as in $\mathrm{NH}_{3} / \mathrm{pH}_{2}$ studies. ${ }^{14}$ Thus, the RVD procedure was typically performed even more rapidly by setting $\Phi_{H_{2}} \approx 400 \mathrm{mmol} \mathrm{hr}^{-1}$ to enhance observations of metastable propyne. Rapid-scan FTIR spectra recorded with modest time resolution (3-5 min depending on averaging) were collected during the deposition step while calibrating the dopant flowrate with $\Phi_{\mathrm{H}_{2}}$ in order to enhance $\mathrm{S} / \mathrm{N}$ and maximize contributions from freshly-deposited rotating propyne molecules, and, of course, immediately post deposition 
in order to monitor propyne NSC in as-deposited solid $\mathrm{pH}_{2}$ samples near $\sim 1.7 \mathrm{~K}$ until equilibration. Equilibrium was achieved when intensity changes between difference spectra were deemed undetectable, which was optimally on the order of the effective time constant of propyne NSC, that is to say, relaxation periods of $\delta t \approx 300$ min or greater.

Important experimental parameters for each experiment in this study are summarized in the Supporting Information, Table S1, including optical setups, instrument resolution, $\mathrm{H}_{2}$ flow rates, $\mathrm{pH}_{2}$ sample thicknesses, hcp fractions, propyne concentrations and the IR bands used to determine them, and the duration of the relaxation period for each NSC kinetic run.

\section{RESULTS AND DISCUSSION}

Propyne $\left(\mathrm{CH}_{3} \mathrm{C} \equiv \mathrm{CH}\right)$ is the simplest linear hydrocarbon containing a methyl rotor. The gasphase spectroscopy of this prolate symmetric top $\left(A^{\prime \prime}=5.31 \mathrm{~cm}^{-1}, B^{\prime \prime}=0.29 \mathrm{~cm}^{-1}\right)^{34}$ has been extensively studied as a model system to study vibrational energy flow in molecules. ${ }^{34-37}$ Belonging to the $C_{3 v}$ point group, propyne has ten fundamental vibrational modes with half of the modes, $v_{1}-v_{5}$, having $A_{1}$ symmetry, while the other half, $v_{6}-v_{10}$, are degenerate with $E$ symmetry. ${ }^{38,39}$ Accordingly, parallel $(\Delta K=0)$ and perpendicular transitions $(\Delta K= \pm 1)$ of propyne are expected for the $A_{1}$ and $E$ symmetry vibrations, respectively. As discussed previously, the end-over-end tumbling motion of propyne will likely be quenched similar to acetylene ${ }^{19}$ when isolated in solid $\mathrm{pH}_{2}$, but the spinning rotation of the methyl group, corresponding to the $A$ rotational constant, should continue to freely rotate similar to $\mathrm{CH}_{3} \mathrm{~F} .{ }^{21}$ Based on van der Waals radii and the structure of propyne, ${ }^{34}$ propyne occupies a double substitution site in solid $\mathrm{pH}_{2}$ with cylindrical symmetry. Therefore, for propyne isolated in solid $\mathrm{pH}_{2}$, we expect that the $J$ and $M_{J}$ rotational quantum numbers appropriate for the gas-phase molecule in free space are lost, and only the $K$ rotational quantum number survives. Basically, the three-dimensional rotational 
motion of propyne in free space transforms into one-dimensional rotation like a particle-on-aring, and two angular librational degrees of freedom, upon suspension in the double substitution site. Therefore, we break from using conventional gas-phase spectroscopic notation and instead use a simplified notation, namely, $\Delta K_{v^{\prime}}\left(K^{\prime \prime}\right)$, where $\Delta K=-1,0,+1$ are designated by $P, Q, R$ and $v^{\prime}$ and $K^{\prime \prime}$ are the vibrational quanta in the upper state and the lower state $K$ quantum number.

Each $K$ rotational state is entangled with a single nuclear spin wavefunction of a specific symmetry to satisfy the symmetrization postulate of quantum mechanics. For $\mathrm{CH}_{3} \mathrm{CCH}$ with three identical hydrogen nuclei (fermions), the ortho $\left(\Gamma_{n s}=A_{1}, I=3 / 2\right)$ nuclear spin wavefunctions combine with $K=0,3,6, \ldots$ rotational states in the ground vibrational state and para $\left(\Gamma_{n s}=E\right.$, $I=1 / 2$ ) nuclear spin wavefunctions combine with $K=1,2,4,5, \ldots$ rotational states. Thus, at the high temperature limit $(T>50 \mathrm{~K})$ a gaseous sample of propyne consists of roughly 50:50 mixtures of the ortho and para spin isomers because the factor of two greater nuclear spin weight for the ortho spin isomer cancels the factor of two more $K \neq 3 n$ rotational states of the para isomer.

For $A_{1}$ symmetry vibrations, parallel transitions should lead to single peaks which correspond to overlapping $Q_{1}(0)$ and $Q_{1}(1)$ transitions out of the lowest rotational states for the ortho and para nuclear spin isomers, respectively. The fact that we observe $Q_{1}(0)$ transitions (vide infra) is another observable in support of the fact that end-over-end rotation of propyne is quenched; in the gas-phase no $Q$-branch is observed for the $K=0$ rotational sub-band. ${ }^{38,39}$ To the extent that the $A$ rotational constant is the same in the upper and lower vibrational states, these transitions will overlap perfectly. However, if $A^{\prime}-A^{\prime \prime} \neq 0$, then the $Q_{1}(0)$ and $Q_{1}(1)$ transitions will occur at slightly different wavenumbers $\left(Q_{\nu^{\prime}}(1)-Q_{v^{\prime}}(0)=A^{\prime}-A^{\prime \prime}\right)$ and there is a possibility of resolving this $K$ fine structure. In contrast, $E$ type vibrations result in perpendicular bands 
with well-resolved $K$ sub-bands that correspond to the $R_{1}(0)$ transition for the ortho nuclear spin modification and $P_{1}(1)$ and $R_{1}(1)$ transitions for the para nuclear spin isomer. For degenerate $E$ vibrations the vibrational angular momentum $l=v_{i}, v_{i}-2, \ldots,-v_{i}$ in the upper state can align or oppose the angular momentum of the $K$ rotation. ${ }^{38,39}$ This gives rise to first-order Coriolis splittings that can be modeled by the energy term $\pm 2(A \zeta)_{v} K l$, where $\zeta$ is the Coriolis coupling constant. Given that the ground state $A^{\prime \prime}$ rotational constant is $\sim 5.31 \mathrm{~cm}^{-1}$ for propyne in the gasphase, ${ }^{34}$ at $1.7 \mathrm{~K}$, transitions out of the $K^{\prime \prime}=2$ rotational state at approximately $21.2 \mathrm{~cm}^{-1}$ ought to be extremely weak due to lack of population of this level. However, if the $K=2$ level is significantly shifted to lower energies, then transitions out of this level may also be observed.

3A. Infrared Spectroscopy of Propyne in Solid $\mathbf{p H}_{2}$. One of the main difficulties in making detailed assignments of the infrared spectrum of propyne isolated in solid $\mathrm{pH}_{2}$ is the fine structure observed for some of the vibrational modes. This fine structure can be caused by single-axis $K$ rotation, but can also be due to a variety of causes, such as propyne-( $\left(\mathrm{oH}_{2}\right)_{n}$, clusters, and one wants a way to selectively identify rovibrational transitions of freely rotating propyne monomers. If the goodness of the $K$ rotational quantum number is lost due to clustering or trapping in defect sites, then we cannot differentiate between different nuclear spin isomers using rovibrational spectroscopy. With this in mind, we begin with the assignment of the $A_{1}$ vibrational modes of propyne.

As discussed above, transitions involving $A_{1}$ vibrational modes should be observed as single peaks (or two overlapping peaks) close to the gas-phase vibrational origins. Typically, $\mathrm{pH}_{2}$ matrix shifts ${ }^{1-4}$ are less than $1 \%$ and, thus, for well-resolved single peaks, vibrational assignments are made simply by comparing the measured peak wavenumber with a potential gas-phase origin. Shown in Figure 1(a) are absorption spectra from 750 to $1500 \mathrm{~cm}^{-1}$ for a 2.5(2) 
mm thick, 200(39) ppm propyne/ $\mathrm{pH}_{2}$ sample recorded at $1.70(1) \mathrm{K}$ at 2.63 (red) and $491 \mathrm{~min}$ (blue) after deposition. The spectra are intentionally offset from zero to show the difference spectrum. All the $A_{1}$ vibrational modes in this region are easily assigned via comparison with the vibrational origins reported in the literature and these $A_{1}$ vibrational assignments are presented in Table 1. However, by constructing difference spectra recorded at short and long time delays after deposition, we can try to resolve these two bands based on NSC. RVD of room temperature propyne into solid $\mathrm{pH}_{2}$ at $1.7 \mathrm{~K}$ kinetically traps the higher energy para nuclear spin isomer in the $K=1$ rotational state due to slow NSC relative to vibrational and rotational relaxation. Assuming that NSC is slow albeit measurable, then by constructing difference $\operatorname{spectra}^{40}$ as $\left(A_{t}-A_{\infty}\right)$ as a function of time ( $t=0$ defined as the end of deposition) allows us to identify absorptions due to the ortho and para nuclear spin isomers of propyne. On the basis of the $+/$ - phase of the peak in the difference spectrum, positive peaks are due to the para spin isomer and will be maximum at $t=0$ and must decrease with time. Negative peaks are minimum at $t=0$ and increase to a maximum at $t=\infty$ (i.e., $\approx 4 \mathrm{~h}$ ) as population is transferred to the lower energy $K=0$ rotational state of the ortho nuclear spin isomer. Furthermore, peaks in the difference spectra can be used to selectively identify absorptions due to propyne monomers that are undergoing unhindered $K$ rotation. As we will show, some peaks in the absorption spectra do not appear in the difference spectra and this obviously inhibits the para and ortho assignments of these peaks. Nevertheless, peaks with sufficient intensity in the difference spectra, that exhibit quantitatively the correct NSC kinetics, allow the nuclear spin isomer responsible for that transition to be rigorously assigned.

Careful inspection of the difference spectrum shown in Figure 1(b) illustrates our strategy for making detailed assignments. For $A_{1}$ symmetry parallel bands $(\Delta K=0)$, the wavenumber 
difference in the $Q_{1}(0)$ and $Q_{1}(1)$ sub-bands must be comparable to the FWHM of the individual absorptions to detect a difference signal. All the $A_{1}$ vibrational bands shown in Figure 1(a) do not show strong difference signals. Based on gas phase measurements, ${ }^{41,42}$ detecting difference signals for both the $v_{5}$ and $v_{4}$ fundamentals should be difficult because the $A^{\prime}-A^{\prime \prime}$ differences are small, $-0.0073 \mathrm{~cm}^{-1}$ and $0.017 \mathrm{~cm}^{-1}$, respectively, and both peaks have modest integrated intensities (see Supporting Information, Table S2). Obviously, small population differences are detected with higher sensitivity using absorptions with large integrated intensities. The $2 v_{9}{ }^{0}$ overtone peak at $1254.47 \mathrm{~cm}^{-1}$ has a large integrated intensity ${ }^{43}\left(16.81(29) \mathrm{km} \mathrm{mol}^{-1}\right)$, but again based on gas phase measurements ${ }^{44}$ the $A^{\prime}-A^{\prime \prime}=-0.00292 \mathrm{~cm}^{-1}$ value is quite small. The small difference signal for the $2 v_{9}^{0}$ overtone peak shown in Figure 1(b) therefore shows that the $Q_{2}(0)$ and $Q_{2}(1)$ absorption features are strongly overlapped making it difficult to determine the splitting. Thus, all the $A_{1}$ vibrational modes displayed in Figure 1 do not show a clear difference spectrum and are assigned to overlapping $Q_{1}(0) / Q_{1}(1)$ peaks. However, as we will show for $v_{2}$, when the $A^{\prime}-A^{\prime \prime}$ difference becomes a little larger, a clear difference signal can be measured for $A_{1}$ type vibrations that provide information on the vibrational origin and the value of $A^{\prime}-A^{\prime \prime}$ for a particular vibrational mode.

For $E$-type vibrational symmetry modes that produce perpendicular bands $(\Delta K= \pm 1)$, the rovibrational transitions for the para and ortho spin isomers are spectrally resolved making assignments rather straightforward. For example, also shown in Figure 1 is the $v_{7}$ (see Supporting Information, Figure S5) absorption of propyne near $1450 \mathrm{~cm}^{-1}$. This mode shows a number of peaks in absorption, but the difference spectrum reveals a positive peak at 1443.54 $\mathrm{cm}^{-1}$ and a negative peak at $1455.12 \mathrm{~cm}^{-1}$ for the para and ortho nuclear spin isomers, respectively. These nuclear spin assignments are rigorous, and we can therefore tentatively 
assign these two peaks to the $P_{1}(1)$ and $R_{1}(0)$ transitions of the para and ortho nuclear spin isomers, respectively. The energy difference between these two transitions predicted using the gas-phase rotational constants ${ }^{41}$ (neglecting centrifugal distortion) is $14.45 \mathrm{~cm}^{-1}$, which compares favorably to the measured value of $11.58 \mathrm{~cm}^{-1}$ and is also consistent with assigning the higher wavenumber transition to the ortho nuclear spin isomer, as indicated by the difference spectrum. Similarly, the $v_{8}$ mode shows (see inset in Figure $1 b$ ) positive and negative difference spectra peaks that are assigned to the para $P_{1}(1)$ and ortho $R_{1}(0)$ peaks, respectively. These two rovibrational transitions are typically the strongest for $E$-type vibrational modes and the $R_{1}(1)$ transition is also observed sometimes, but is usually much broader and harder to detect. The rovibrational assignments of $E$ type vibrational bands are also listed in Table 1. In what follows, we examine various propyne bands in greater detail in order to articulate some of the challenges associated with making detailed rovibrational assignments.

After assigning ortho and para nuclear spin symmetries to the various peaks present in the spectrum of propyne using the difference spectra, we can attempt $K$ rotational assignments using conventional energy levels expressions developed for gas-phase prolate symmetric tops. ${ }^{38,39}$ However, since we expect that $J$ is no longer a good quantum number, the energy level expressions are modified to consider only terms associated with single-axis $K$ rotation (neglecting centrifugal distortion). In the Supporting Information, Section 2 various expressions are discussed for $Q, R$, and $P$ transitions with $K^{\prime \prime}=0,1$, and 2 for both parallel $\left(A_{1}\right)$ and perpendicular $(E)$ rovibrational bands. This information is used to compare the measured transition wavenumbers of propyne with gas-phase results to attempt detailed assignments.

The first band investigated in detail was the $v_{2}$ (symmetric methyl $\mathrm{CH}$ stretch) fundamental near $2941 \mathrm{~cm}^{-1}$. This vibrational mode has a large integrated band strength (see 
Supporting Information, Table S2) and therefore is sensitive to small population differences. As we will show, this band can be used to effectively measure the NSC kinetics of propyne in solid $\mathrm{pH}_{2}$. The $v_{2}$ spectrum recorded at $0.02 \mathrm{~cm}^{-1}$ resolution is shown in Figure 2. Spectra recorded 5.08 and 291.8 min after deposition are shown in Figure 2(a) as red and blue traces, respectively. Both spectra are intentionally offset from zero to better show the resulting difference spectrum. Shown in Figure 2(b), therefore, is the difference spectrum. The difference spectrum displays the signature lineshape for overlapping $Q_{1}(1)$ and $Q_{1}(0)$ transitions for the two nuclear spin isomers, namely, the positive $Q_{1}(1)$ peak is assigned to the para isomer and the negative $Q_{1}(0)$ peak to the ortho isomer. Based on the gas-phase rotational constants, ${ }^{35,45}$ these two transitions are predicted ${ }^{35}$ to be split by $0.055 \mathrm{~cm}^{-1}$ (or 0.090 based on Ref. 45) with the $Q_{1}(0)$ transition of the ortho nuclear spin isomer at higher wavenumbers. For propyne isolated in solid $\mathrm{pH}_{2}$, we observe these two bands with the same energetic ordering as measured in the gas-phase, and with a splitting of around $0.11 \mathrm{~cm}^{-1}$. These measurements demonstrate that the change in the $A$ rotational constant upon $v_{2}$ excitation is very small, but does not provide information on the value of $A$ in either the ground or excited vibrational states. It also demonstrates how the difference spectrum of a parallel band can be used to measure the growth and decay in the populations of the two nuclear spin isomers of propyne. By definition, the difference spectrum is only sensitive to propyne populations that undergo $K$-rotation and NSC and therefore rejects overlapping propyne features that do not contribute to NSC. For example, careful examination of Figure 2 reveals that the total $v_{2}$ lineshape is not well represented by the sum of two overlapping pseudo-Voigt peaks, but rather this absorption has a shoulder to the low wavenumber side $\left(\sim 2934.4 \mathrm{~cm}^{-1}\right)$ that does not change in intensity with time. In addition, integration of the entire difference spectrum results in a value of zero indicating that the line 
strengths for the two transitions are approximately equal. This is consistent with both these transitions being pure vibrational transitions (not rovibrational) and the $Q_{1}(0)$ transition has the same integrated band strength as the $Q_{1}(1)$ peak. This is a direct ramification of the loss of $J$ as a good quantum number such that the $Q_{1}(0)$ transition is possible for the matrix isolated propyne.

In Figure 3, we present the full spectrum of $v_{6}$ (antisymmetric methyl $\mathrm{CH}$ stretch) which is representative of a perpendicular band. ${ }^{35,46}$ Here we see more deviations from gas-phase behavior, signaling perturbations in the $K$ rotational motion. For the absorption spectra recorded at short and long times after deposition, we measure approximately 7 peaks excluding the two very weak peaks at the extremes of the spectral range displayed in Figure 3. However, in the difference spectrum, three peaks are absent (marked with asterisks in Fig. 3) and we only have clear difference signals for four peaks, indicating that the three central peaks shown in Figure 3 do not show the same NSC kinetics as the others. It is possible that these peaks are due to propyne- $\left(\mathrm{oH}_{2}\right)_{n}$ clusters that form irreversibly due to the stronger attractive intermolecular forces between propyne and $\mathrm{oH}_{2}$ compared to $\mathrm{pH}_{2}$; however, assignment of these cluster peaks extends beyond the scope of this work. Based on the idea that the strongest transitions should be out of the $K=0$ and 1 rotational states, we should observe one $R_{1}(0)$ peak for the ortho nuclear spin isomer, and two transitions for the para isomer, namely, $P_{1}(1)$ and $R_{1}(1)$. We do observe two peaks for the para spin isomer, but we also observe two $R_{1}(0)$ peaks for the ortho spin isomer.

As first demonstrated by Lee, $\mathrm{Wu}$ and Hougen, ${ }^{21}$ we can attempt to make $K$ rotational assignments of the $v_{6}$ band based on a simple energy level expression for single-axis $K$ rotation and the molecular constants measured in the gas-phase (see Supporting Information, Section 2). To begin, we make the simplifying assumption that we can use the average of the two observed $R_{1}(0)$ transitions for the $R_{1}(0)$ transition wavenumber $\left(R_{1}(0)_{\text {avg }} \approx 2979.7 \pm 0.7 \mathrm{~cm}^{-1}\right)$. As we will 
show, it doesn't matter how we treat this obvious deviation from gas-phase behavior, we cannot extract a consistent set of molecular constants. We first assigned the three rovibrational transitions shown in Fig. 3 corresponding to $K^{\prime \prime}=0,1$ and assuming the gas-phase Coriolis constant $\left(\zeta_{6}=0.07250\right)^{46}$ is preserved, we determined the three molecular constants presented in Table 2. This gave reasonable results consistent with reduced $A$ constants for propyne $K$-rotation in solid $\mathrm{pH}_{2}$. However, next, we used all 5 rovibrational transitions labeled in Figure 3 corresponding to $K^{\prime \prime}=0,1,2$. Whereas the assignment of $K^{\prime \prime}=2$ transitions seems beyond a doubt, by comparison with gas phase values (Table 1$)$, the relation $R_{1}(2)-P_{1}(2)=2\left[R_{1}(1)-\right.$ $\left.P_{1}(1)\right]$, deduced from the simplified model, is not fulfilled, independent of the value of the Coriolis constant. All variations of the fitting procedure given in the Supporting Information, Section 2, fixed and floated $\zeta_{6}$, and including a $D_{K}$ distortion constant resulted in fits that could not reproduce the transition wavenumbers to within experimental precision (e.g., $r m s=1.2 \mathrm{~cm}^{-1}$ ). This is clearly a statement that the measured spectra, assigned via the methods described, do not readily conform to expressions determined for single-axis $K$ rotation. Thus, comparison of the determined molecular constants with gas-phase values to make detailed $K$ rotational assignments is likely misleading, especially when assumptions must be made given a limited number of transitions. This is an intrinsic problem with conducting these types of rovibrational assignments that only involve a few transitions and therefore lack built-in redundancies in the spectrum (such as four line combination differences), there is no way to rigorously test potential assignments. Surely, qualitative insights can be ascertained, but true predictive understanding of the energy patterns can only be advanced by comparing with fully quantum mechanical simulations that include interactions of the dopant with the surrounding $\mathrm{pH}_{2}$ solid. 
The $E$ type $v_{7}\left(\mathrm{CH}_{3}\right.$ skeletal deformation) vibrational mode at roughly $1450 \mathrm{~cm}^{-1}$, shown previously in Figure 1, was also studied in detail (see Supporting Information, Figure S5 for the expanded spectrum). For this band, we only observed the $P_{1}(1), R_{1}(0)$, and $R_{1}(1)$ transitions and, thus, to extract molecular constants from this mode we had to assume that the gas-phase Coriolis coupling constant $\left(\zeta_{7}=-0.31424\right)^{41}$ is largely unchanged upon solvation in solid $\mathrm{pH}_{2}$. Similar to the $v_{6}$ band, the $R_{1}(0)$ peak consists of two partially resolved peaks and therefore we used the average peak position $\left(R_{1}(0)_{\mathrm{AVG}}=1454.9 \mathrm{~cm}^{-1}\right)$. There is some doubt in the assignment of the $R_{1}(1)$ transition, either to a feature at 1458.4 or $1473.88 \mathrm{~cm}^{-1}$. However, using either $R_{1}(1)$ assignment, the simplified model with the gas phase Coriolis coupling constant leads to unphysical $A$ rotational constants, and importantly, to a different $A^{\prime \prime}$ constant than the $v_{6}$ analysis. The three molecular constants determined from this analysis with $R_{1}(1)$ at $1473.88 \mathrm{~cm}^{-1}$ are presented in Table 2. Once again, this analysis demonstrates that quantitative analysis of the extracted molecular constants from $K$-rotational assignments of only three transitions is potentially misleading. The only other $E$ type vibration where this analysis is possible is the $v_{3}+v_{8}$ combination band and the results are also presented in Table 2. Clearly, the analysis of each band leads to a different $A^{\prime \prime}$ constant each time. The fact that this type of analysis does not produce a consistent ground state $A^{\prime \prime}$ constant implies that either the assumption that the Coriolis constant does not change, or the assumed energy level expression, is incorrect. Applying a similar type of analysis to the three $E$-type rovibrational bands reported in the literature ${ }^{21}$ for $\mathrm{CH}_{3} \mathrm{~F}$ in solid $\mathrm{pH}_{2}$ leads to a similar conclusion; each band produces a different ground state $A^{\prime \prime}$ rotational constant. This suggests that the $K$-rotational energy levels of both methyl rotor molecules, $\mathrm{CH}_{3} \mathrm{CCH}$ and $\mathrm{CH}_{3} \mathrm{~F}$, are not well described by simple single-axis $K$ rotational energy level expression. 
We repeatedly observed two $R(0)$ lines for many of the $E$-type propyne bands $\left(v_{6}, v_{7}, v_{8}\right.$, $2 v_{6}$ ). This is most likely due to the selection rules for $C_{3 v}$ symmetric tops, the fact that Coriolis perturbations are possible in upper degenerate vibrational states, and the loss of the $J$ quantum number for propyne isolated in solid $\mathrm{pH}_{2}$. Neither of the rotational levels connected by the $P_{1}(1)$ rovibrational transition are effected by Coriolis perturbations and the $R_{1}(1)$ line connects $E$ symmetry rotational levels. ${ }^{47}$ But the $R_{1}(0)$ transition accesses the $K=1$ rotational state in the upper vibrational state and thus in the gas-phase accesses alternating $A_{1}$ and $A_{2}$ rotational states as a function of $J$. In the present case where the $J$ quantum number is lost, Coriolis perturbations of the upper rotational state could produce two spectrally resolved $R_{1}(0)$ transitions. Furthermore, observation of two $R_{1}(0)$ transitions also contributes to the difficulty of extracting consistent molecular constants from the analysis of the $P_{1}(1), R_{1}(0)$, and $R_{1}(1)$ transitions. Thus, it appears that the vibrational angular momentum generated in the upper degenerate vibrational state that is coupled to the $K$-rotation produces shifts in the transition wavenumbers on the order of the $K$ sub-band spacing. Two $R_{1}(0)$ transitions could also appear when considering a removal of the degeneracy of the upper $E$ vibrational state due to the solid environment. If the perturbation due to the solid environment is large enough to induce torsional barriers, the $K^{\prime \prime}=0$ and 1 levels are better represented by $A$ and $E$ levels, respectively, in the periodic torsional potential (splitting due to $\mathrm{H}$ atom tunneling) as was described in Ref. 23 in the case of the acetylacetone molecule. The analysis of the NSC process remains the same, but the structure presented in the spectrum by the $P_{1}(1)$ and especially the $R_{1}(1)$ transitions is difficult to assign.

These same types of discrepancies are not found in the interpretation of rovibrational spectra of molecules isolated in superfluid He nanodroplets. ${ }^{5-9}$ Typically, the measured rotational constants for molecules dissolved in He nanodroplets are reduced $(39 \%$ and $95 \%$ for 
heavy and light rotors) from gas-phase values, and distortion constants can be anomalously large, but the standard spectroscopic signatures are preserved along with the selection rules based on the symmetry of the gas-phase transition dipole moment. ${ }^{5-9}$ For example, the parallel $(\Delta K=0) v_{1}$ spectrum (acetylenic C-H stretch) of propyne was recently revisited for propyne isolated in $\mathrm{He}$ nanodroplets. ${ }^{48,49}$ The spectrum displays $P, Q$, and $R$ branches appropriate for a $C_{3 \mathrm{v}}$ symmetric top molecule. Comparison between the fitted constants for gas-phase propyne and propyne solvated in He nanodroplets shows that the $B$ rotational constant is reduced to $26.0(1) \%$ and $25.6(1) \%$ of its gas-phase value for the ground and $\mathrm{v}=1$ excited vibrational states, respectively, and the $\Delta_{J^{\prime \prime}}$ distortion constant increases by a factor of 5100 compared to the gas-phase. However, using these effective rotational constants, the spectrum is relatively well reproduced demonstrating that the selection rules are preserved. ${ }^{49}$ Note that the $v_{1}$ band is a parallel band so that the spectrum is not sensitive to the $A$ constant directly, but rather the difference in $A$ constants with vibrational excitation. Unfortunately, the corresponding $v_{1}$ spectrum for propyne isolated in solid $\mathrm{pH}_{2}$ is not very informative because the difference spectrum shows multiple positive and negative peaks (see Supporting Information, Figure S6). Nonetheless, it is clear that isolation of propyne in solid $\mathrm{pH}_{2}$ perturbs the rotational motion in a qualitatively different way; there is no end-over-end rotation in solid $\mathrm{pH}_{2}$ while in liquid ${ }^{4} \mathrm{He}$ the end-over-end rotational motion is preserved (although greatly hindered) and so are the selection rules. This means that the rovibrational dynamics of molecules in solid $\mathrm{pH}_{2}$ are more difficult to assign rigorously and therefore also harder to interpret. While both $\mathrm{He}$ and $\mathrm{pH}_{2}$ are quantum hosts, one is a liquid and the other a solid and this leads to very different rotational dynamics of guest molecules. In solid $\mathrm{pH}_{2}$, the rotational dynamics are significantly perturbed, end-over-end rotational is quenched, and the selection rules are altered (no $Q$-branch for $K=0$ in the gas-phase), such that the observed 
infrared spectra of molecular dopants isolated in solid $\mathrm{pH}_{2}$ exhibiting $K$-axis rotation are not so easily assigned via comparison to gas-phase spectra. However, the spectra assigned via the methods used here provide new data sets that can be modeled using quantum mechanical dynamic calculations to learn more about the $\mathrm{pH}_{2}$ cage dynamics.

3B. Nuclear Spin Conversion Kinetics. We initiate our analysis of the NSC kinetics of propyne in solid $\mathrm{pH}_{2}$ using the $v_{2}$ vibrational band near $2935 \mathrm{~cm}^{-1}$. As described above, since $v_{2}$ is a parallel band, peaks associated with separate ortho and para nuclear spin isomers are not observed directly. Therefore, difference spectra recorded at various times after deposition are utilized to measure the effective rate constant for para-to-ortho NSC of propyne. As described in detail in the Supporting Information, Section 1, each difference spectrum is fit to a sum of two normalized symmetric pseudo-Voigt profiles ${ }^{50}$ in order to quantify time-dependent intensity changes in the two overlapping absorptions that are resolved in the difference spectrum. The two difference peaks in Figure 2 can be assigned to the para $(K=1, I=1 / 2)$ and ortho $(K=0, I=3 / 2)$ nuclear spin isomers. A representative difference spectrum for $v_{2}$ fit to the sum of two pseudoVoigt lineshape functions is shown in Figure 4; the difference spectrum is well modeled by this functional form allowing a number of parameters to be extracted from each spectral trace (see Supporting Information, Section 1 for more details). The strength of this procedure is that the peak areas of components in an overlapping band, which are conserved quantities, are extracted directly from the analysis in order to study their temporal behavior. It turns out that the time dependence of the two components in the $v_{2}$ difference spectra are well-fit to solutions of firstorder kinetics equations, where the effective lifetime of propyne NSC, $\tau_{\text {eff, }}$ is statistically equivalent for both components to within the standard errors of the fitted parameters (see Supporting Information, Table S4). 
Representative kinetic traces for the $v_{2}\left(A_{1}\right)$ vibrational mode of propyne are shown in Figure 5a. As described earlier, the red and blue data points represent the para and ortho peak areas determined from fits to the difference spectra. The lines represent the results of leastsquares fits of the data to first-order kinetics equations (see below). Note that the way we calculate the difference spectra means that both peak areas should decay to zero as the propyne nuclear spin populations come into equilibrium at long times after deposition. For the data shown in Figure 5a, we did not wait long enough to achieve equilibrium as evidenced by the crossing of the fits above and below zero. However, this does not affect the determined $\tau_{\text {eff }}$ time constant due to the nature of first-order kinetics. Individual time constants are determined for each peak and are well matched. Repetition of this analysis in four separate experiments yields highly reproducible results (see Supporting Information, Table S4). Examination of Figure 5a also shows that the two peak areas are symmetrically displaced around zero indicating that the line strengths associated with these two peaks are comparable, although the peak FWHM's differ (see Fig. 2).

The $v_{6}$ and $v_{7}$ perpendicular bands were also analyzed to determine effective NSC time constants for propyne. To quantify the NSC kinetics, the $v_{6}$ and $v_{7}$ bands are broken into segments; fortunately, there is enough separation between the various peaks to integrate the peaks corresponding to ortho $\left(R_{1}(0)\right)$ and para $\left(P_{1}(1)\right.$ and $\left.R_{1}(1)\right)$ nuclear spin isomer populations. The integrated intensities are well-fit to first-order kinetic equations given by

$$
\begin{aligned}
& I_{\text {para }}(t)=I_{\text {para }}(\infty)+\left(I_{\text {para }}(0)-I_{\text {para }}(\infty)\right) \exp (-t / \tau) \\
& I_{\text {ortho }}(t)=I_{\text {ortho }}(0)+\left(I_{\text {ortho }}(\infty)-I_{\text {ortho }}(0)\right)[1-\exp (-t / \tau)]
\end{aligned}
$$

in which the fitted lifetimes agree statistically with the $v_{2}$ data to within error (Supporting Information, Table S4). Representative kinetic traces for $v_{6}$ and $v_{7}$ are shown in Figure $5 b$ and 
5c, respectively. The kinetic traces in Fig. $5 \mathrm{~b}$ and $5 \mathrm{c}$ are different from $5 \mathrm{a}$ because they were constructed from the integration of absorption spectra, not difference spectra. We did check that the total integrated intensity of each band remains constant over the time window of the kinetic analysis. Eleven separate kinetic analyses of the $v_{2}, v_{6}$ and $v_{7}$ bands of propyne all agree with each other and produce a weighted average time constant of $\tau_{\text {eff }}=287(7) \mathrm{min}$.

As a further test of this kinetic analysis, we also fitted the difference spectrum of the $v_{2}$ mode of ${ }^{13} \mathrm{CH}_{3}{ }^{12} \mathrm{C}_{2} \mathrm{H}$ present in natural abundance $(\sim 1.1 \%)$ for the experiment with the highest propyne concentration. The other ${ }^{13} \mathrm{C}$ isotopologs likely overlap with the $v_{2}$ mode of the most abundant isotopolog. ${ }^{35,51}$ As expected, this peak was also well-fit by expressions (Eq. 2 and 3) and gave a time constant of $\tau_{\text {eff }}=170(20)$ min, which is approximately 1.7(3) times faster than the value determined for the ${ }^{12} \mathrm{C}_{3} \mathrm{H}_{4}$ isotopomer. The reason for the faster $\mathrm{NSC}$ in ${ }^{13} \mathrm{CH}_{3}{ }^{12} \mathrm{C}_{2} \mathrm{H}$ is likely because the ${ }^{13} \mathrm{C}(I=1 / 2)$ nucleus interacts with the $\mathrm{H}$-atoms on the methyl group via magnetic dipole-dipole coupling which introduces an additional relaxation channel not open to the normal isotopolog. ${ }^{52-54}$ It would be interesting to put the ${ }^{13} \mathrm{C}$ on the acetylenic carbons to see if the time constant increases for the more remote atom with non-zero nuclear spin.

\section{C. Measuring the para-to-ortho ratio for propyne in solid $\mathrm{pH}_{2}$ at low temperature.}

The thermodynamic equilibrium concentrations for the para and ortho nuclear spin isomers of propyne are given by the respective rotational partition functions as a function of temperature. This para-to-ortho ratio $(P O R)$ is defined here as the equilibrium constant for the reversible, first-order nuclear spin conversion process between the two spin isomers of propyne, namely,

$$
\text { ortho-propyne } \leftrightarrow \text { para-propyne; POR }=\frac{[\text { para }]}{[\text { ortho }]}
$$

As discussed earlier, in the high temperature limit the POR approaches unity, whereas this ratio goes to zero at $T \rightarrow 0 \mathrm{~K}$. We were therefore interested in calculating the experimental $P O R$ 
measured in these studies after the system has come into equilibrium as another way to determine the energy splitting between the $K=1$ and $K=0$ rotational states. However, in order to do this necessitates that we transform our integrated intensities into relative populations, which means the line strengths of individual rovibrational transitions must be determined. In calculating propyne concentrations using Eq. (1) we simply integrate over the entire band and use the integrated IR band intensities given in the Supporting Information, Table S2, but if we want rotational populations we must deal with rovibrational line strengths. To do this rigorously is difficult, thus, we used an approximation that has been used by others, ${ }^{55}$ where we assume that the populations in the $K=0$ and $K=1$ follow this equation, $n_{K=1}+n_{K=0}=1$, that is, that the system is well described as a two component system, then the relative line strengths can be determined from intensity-intensity correlation plots. ${ }^{55}$ Correlation plots of the intensities for various transitions in $v_{6}$ can be generated as shown in the Supporting Information, Figure S9 and Table S5. Furthermore, this approach allows us to estimate the $P O R$ as a function of time during an experiment and also to estimate the energy of the $K=1$ excited rotational state of the para nuclear spin isomer.

We analyzed the $v_{6}$ spectra for six different experiments in terms of the $P O R$ to see if general trends could be quantified. A representative POR trace is shown in Figure 6. As stated earlier, the high temperature statistical limit is $P O R=1.0$, where there is an equal mixture of ortho and para nuclear spin isomers. Upon RVD of room temperature propyne gas into solid $\mathrm{pH}_{2}$, the initial $P O R \approx 0.9$ at $\sim 2.5 \mathrm{~K}$ during deposition. By the end of deposition $(t=0)$, the $P O R$ drops to a value of $\sim 0.75$ and after deposition it continues to decay at $1.7 \mathrm{~K}$. From a fit to the experimental data, the $P O R$ decays to an equilibrium value of $\sim 0.17$. This is an order of magnitude greater than the theoretical limit predicted using the gas-phase rotational energies at 
equilibrium at $1.7 \mathrm{~K}(P O R=0.0117)$ and therefore signals a lowering of the $K=1$ level. We note that this analysis does suffer from potential systematic errors due to contributions of the nonrotating propyne absorption at $2978.29 \mathrm{~cm}^{-1}$ to the integrated intensity of the $R_{1}(0)$ peak, the fact that there are two $R_{1}(0)$ peaks for $v_{6}$, and errors in the measured temperature. However, given the difficulties in determining $A^{\prime \prime}$ spectroscopically, this might be the best way to measure the ground state $K=1$ and $K=0$ energy splitting.

Using this approach, we estimate the energy difference between the lowest para $(K=1)$ and ortho $(K=0)$ rotational states, or in other words the effective $A^{\prime \prime}$ constant. This analysis predicts a value of $2.2(6) \mathrm{cm}^{-1}$ and is compared with the spectroscopic constants in Table 2. This value suggests that propyne $K$-rotation is strongly perturbed. This analysis predicts that the $A^{\prime \prime}$ rotational constant is about $42 \%$ of the gas-phase value and therefore the population of the para nuclear spin isomer at equilibrium at $1.7 \mathrm{~K}$ is significantly greater than what is predicted using the gas-phase rotational constants. Given that we measure an equilibrium $P O R$ value of $\sim 0.15$ at $1.7 \mathrm{~K}$, the approximate temperature where all the experiments were conducted, this implies that the elementary rate constants for the reversible first-order NSC kinetics are significantly different from the measured effective rate constant. ${ }^{56}$ We can use the measured $k_{\text {eff }}$ and $P O R(1.7(1) \mathrm{K}$, $t=\infty$ ) for five experiments (Expt. 3 was discarded) to determine $k_{p \rightarrow o}=3.00(9) \times 10^{-3} \mathrm{~min}^{-1}$ and $k_{o \rightarrow p}=4.4(11) \times 10^{-4} \mathrm{~min}^{-1}$.

Another way therefore to refine the POR determination would be to perform kinetic experiments at different temperatures. We should be able to measure different asymptotic values of POR (at equilibrium) as a function of temperature. All the experiments in the Wyoming laboratory were conducted at the deposition temperature because we did not want to complicate the analysis of the difference spectra by including changes due to annealing or $\mathrm{oH}_{2}$ diffusion, 
which can occur over comparable timescales relative to NSC at elevated temperaures. ${ }^{1-4}$ However, the group in Orsay conducted experiments for samples that were equilibrated for longer times at $2.8 \mathrm{~K}$, and were annealed at $4.0 \mathrm{~K}$ for brief periods of time. The group in Orsay uses a closed-cycle cryostat and thus is capable of monitoring the same sample for days. The group in Wyoming uses a bath cryostat and thus can only hold samples for approximately 10 hours without the need to refill with liquid helium. Spectra from the Orsay laboratory were analyzed using the same procedure and line strengths determined in Wyoming. The measured values from the two laboratories are presented in Figure 7. The lines presented in Figure 7 represent $P O R s$ calculated for single-axis $K$-rotation $\left(E=A^{\prime \prime} K^{2}\right)$ with effective $A^{\prime \prime}$ constants equal to $1.00 \mathrm{~cm}^{-1}, 2.22 \mathrm{~cm}^{-1}$, and $5.31 \mathrm{~cm}^{-1}$, respectively. Error bars on each measurement are calculated using propagation of errors. The data from the two labs are somewhat consistent with greater PORs calculated at higher temperatures in Orsay. All the data lies between the two limiting values of $A^{\prime \prime}=1.00$ and $5.31 \mathrm{~cm}^{-1}$. As can be seen in Figure 7, there is significant scatter in the measured PORs, but the level of agreement is promising given the difficulties in making intensity based measurements. We hope this work motivates similar measurements for different methyl rotor molecules and we want to continue to refine these measurements for propyne by conducting further experiments in both laboratories.

It is interesting to compare the NSC rate constant determined here for propyne with the one measured at $3.3 \mathrm{~K}$ for $\mathrm{CH}_{3} \mathrm{~F}$ isolated in solid $\mathrm{pH}_{2}{ }^{21}$ At $3.3 \mathrm{~K}$, based on our analysis of propyne with $A^{\prime \prime}=2.2(6) \mathrm{cm}^{-1}$, we would predict a $P O R$ of approximately 0.3 which would imply $k_{\text {eff }}(3.3 \mathrm{~K}) \approx 3.8 \times 10^{-3} \mathrm{~min}^{-1}$ as compared to $2.2(5) \times 10^{-3} \mathrm{~min}^{-1}$ for the fast NSC rate constant measured over the first 5 hours for $\mathrm{CH}_{3} \mathrm{~F}$ solvated in solid $\mathrm{pH}_{2}$ at $3.3 \mathrm{~K}^{21}$ Previous measurements of $\mathrm{CH}_{3} \mathrm{~F}$ NSC found bi-exponential behavior that the authors ascribed to fast $\mathrm{oH}_{2}$ 
assisted NSC of $\mathrm{CH}_{3} \mathrm{~F}$, followed by slower $\left(k=3.7(8) \times 10^{-4} \mathrm{~min}^{-1}\right) \mathrm{NSC}$ when the $\mathrm{oH}_{2}$ concentration drops below a critical value. ${ }^{21}$ There is no evidence for bi-exponential behavior in the current NSC kinetics, but measurements over larger time windows would be helpful. Interestingly, the opposite behavior was found for $\mathrm{NSC}_{\text {of }} \mathrm{ClCH}_{2}$ trapped in solid $\mathrm{pH}_{2}$ where the conversion rate decreased by a factor of 1.5 for samples with $10 \% \mathrm{oH}_{2} \cdot{ }^{27}$ All the measurements conducted in Wyoming were only performed for up to 8 hours. Preliminary results on longer timescales performed in Orsay show the time evolution of the three peaks marked with an asterisk in Figure 3 (and Figures S4 and S5), and at much longer timescales, two peaks decrease with time and one increases, meaning that the behavior at long times is more complex. An analysis of these peaks in terms of $\mathrm{oH}_{2}$ clustering or different matrix sites must be performed to go further in this analysis.

\section{CONCLUSIONS}

We present here a detailed spectroscopic analysis of propyne isolated in solid $\mathrm{pH}_{2}$. This analysis shows how NSC can be used to rigorously assign the nuclear spin isomer responsible for a given rovibrational transition, provided that the molecules exhibit single-axis $K$ rotational motion. Further, through the analysis of three separate perpendicular rovibrational bands, a detailed $K$ rotational assignment is not possible that delivers a consistent set of ground state rotational constants. This demonstrates that the rotational levels of propyne solvated in solid $\mathrm{pH}_{2}$ are not well described by simple one-dimensional free rotor expressions, and that quantum calculations that include intermolecular interactions with the quantum host are likely necessary for acceptable agreement with experiment. This data therefore contains detailed experimental information on how the quantum cavity influences the large amplitude rotational motion of a dopant, but the 
complication of how best to extract this information still remains. It is hoped that the procedures used in this study are applicable to a wide variety of molecules containing methyl rotors to permit systematic studies.

The NSC kinetics of propyne in solid $\mathrm{pH}_{2}$ show single-exponential decay indicative of a reversible first-order process. Careful analysis of three separate bands all lead to an average effective time constant of $\tau_{\text {eff }}=287(7) \mathrm{min}$ for NSC at $1.7 \mathrm{~K}$. This time constant is comparable to the fast time constant extracted for $\mathrm{CH}_{3} \mathrm{~F}$ suspended in solid $\mathrm{pH}_{2}$, but as discussed in the previous section, the NSC of propyne should be measured over longer time periods to see if it too shows bi-exponential behavior similar to $\mathrm{CH}_{3} \mathrm{~F}$. Using intensity-intensity correlation plots, we are able to determine the relative line strengths of different propyne rovibrational transitions that allow the POR to be calculated. This data suggests that using the RVD technique we are able to trap $\sim 75 \%$ of the higher rotational energy para nuclear spin isomer immediately post deposition. Further, by measuring the $P O R$ after full thermal equilibration, analysis from both labs suggests that the $A^{\prime \prime}$ rotational constant is significantly reduced from its gas phase value. A reduction in the $A^{\prime \prime}$ rotational constant would be suspected for a hindered rotor in solid $\mathrm{pH}_{2}$ and thus further experiments could better determine this value and hopefully bring the predicted $A^{\prime \prime}$ values estimated from spectroscopic measurements in the two laboratories into better agreement. We plan to study NSC in a series of molecules containing methyl groups in order to see if trends emerge and to further develop these analytical tools and procedures by testing them on a variety of molecules.

\section{ACKNOWLEDGEMENTS}

This work was sponsored in part by the Chemistry Division of the US National Science Foundation (CHE 13-62497). AIS is grateful to the UW Department of Chemistry for providing 
a Summer Research Fellowship during his first summer of graduate school when some of this work was conducted. The Orsay group acknowledges the RTRA Triangle de la Physique (20130436T REACMAQ) for support and the French-Lithuanian PHC GILIBERT program (42125XF and S-LZ-19-1 from RCL). 


\section{REFERENCES}

(1) Bahou, M.; Das, P.; Lee, Y.-F.; Wu, Y.-J.; Lee, Y.-P. Infrared spectra of free radicals and protonated species produced in para-hydrogen matrices. Phys. Chem. Chem. Phys. 2013, $16,2200-2210$.

(2) Momose, T.; Fushitani, M.; Hoshina, H. Chemical reactions in quantum crystals. Int. Rev. Phys. Chem. 2005, 24, 533-552.

(3) Oka, T. High-Resolution Spectroscopy of Solid Hydrogen. Annu. Rev. Phys. Chem. 1993, 44, 299-333.

(4) Yoshioka, K.; Raston, P. L.; Anderson, D. T. Infrared spectroscopy of chemically doped solid parahydrogen. Int. Rev. Phys. Chem. 2006, 25, 469-496.

(5) Callegari, C.; Conjusteau, A.; Reinhard, I.; Lehmann, K. K.; Scoles, G. Superfluid hydrodynamic model for the enhanced moments of intertia of molecules in ${ }^{4} \mathrm{He}$. Phys. Rev. Lett. 1999, 83, 5058-5061.

(6) Callegari, C.; Lehmann, K. K.; Schmied, R.; Scoles, G. Helium nanodroplet isolation rovibrational spectroscopy: Methods and recent results. J. Chem. Phys. 2001, 115, 1009010110.

(7) Grebenev, S.; Toennies, J. P.; Vilesov, A. F. Superfluidity within a small helium-4 cluster: The microscopic Andronikashvili experiment. Science 1998, 279, 2083-2086.

(8) Hartmann, M.; Miller, R. E.; Toennies, J. P.; Vilesov, A. F. High-resolution molecular spectroscopy of van der waals clusters in liquid helium droplets. Science 1996, 272, 1631-1634.

(9) Toennies, J. P.; Vilesov, A. F. Superfluid helium droplets: A uniquely cold nanomatrix for molecules and molecular complexes. Angew. Chem. Int. Ed. 2004, 43, 2622-2648.

(10) Miki, M.; Momose, T. Rovibrational transitions and nuclear spin conversion of methane in parahydrogen crystals. Low Temp. Phys. 2000, 26, 661-668.

(11) Miyamoto, Y.; Fushitani, M.; Ando, D.; Momose, T. Nuclear spin conversion of methane in solid parahydrogen. J. Chem. Phys. 2008, 128, 114502-1-10.

(12) Momose, T. Rovibrational states of a tetrahedral molecule in a hexagonal close-packed crystal. J. Chem. Phys. 1997, 107, 7695-7706.

(13) Tam, S.; Fajardo, M. E.; Katsuki, H.; Hoshina, H.; Wakabayashi, T.; Momose, T. High resolution infrared absorption spectra of methane molecules isolated in solid parahydrogen matrices. J. Chem. Phys. 1999, 111, 4191-4198.

(14) Ruzi, M.; Anderson, D. T. Matrix isolation spectroscopy and nuclear spin conversion of $\mathrm{NH}_{3}$ and $\mathrm{ND}_{3}$ in solid parahydrogen. J. Phys. Chem. A 2013, 117, 9712-9724.

(15) Fajardo, M. E.; Lindsay, C. M. Crystal field splitting of rovibrational transitions of water monomers isolated in solid parahydrogen. J. Chem. Phys. 2008, 128, 014505-1-4.

(16) Fajardo, M. E.; Tam, S.; DeRose, M. E. Matrix isolation spectroscopy of $\mathrm{H}_{2} \mathrm{O}, \mathrm{D}_{2} \mathrm{O}$, and HDO in solid parahydrogen. J. Mol. Struct. 2004, 685-696, 111-127. 
(17) Anderson, D. T.; Hinde, R. J.; Tam, S.; Fajardo, M. E. High-resolution spectroscopy of $\mathrm{HCl}$ and $\mathrm{DCl}$ isolated in solid parahydrogen: Direct, induced, and cooperative infrared transitions in a molecular quantum solid. J. Chem. Phys. 2002, 116, 594-607.

(18) Fajardo, M. E.; Lindsay, C. M.; Momose, T. Crystal field theory analysis of rovibrational Spectra of carbon monoxide monomers isolated in solid parahydrogen. J. Chem. Phys. 2009, 130, 244508-1-10.

(19) Lee, Y.-C.; Venkatesan, V.; Lee, Y.-P.; Macko, P.; Didiriche, K.; Herman, M. Infrared spectra of $\mathrm{C}_{2} \mathrm{H}_{2}$ under jet-cooled and para- $\mathrm{H}_{2}$ matrix conditions. Chem. Phys. Lett. 2007, 435, 247-251.

(20) Tam, S.; Fajardo, M. E. Observation of the high-resolution infrared absorption spectrum of $\mathrm{CO}_{2}$ molecules isolated in solid parahydrogen. Low Temp. Phys. 2000, 26, 653-660.

(21) Lee, Y.-P.; Wu, Y.-J.; Hougen, J. T. Direct spectral evidence of single-axis rotation and ortho-hydrogen-assisted nuclear spin conversion of in solid parahydrogen. J. Chem. Phys. 2008, 129, 104502-1-6.

(22) Lee, Y.-P.; Wu, Y.-J.; Lees, R. M.; Xu, L.-H.; Hougen, J. T. Internal rotation and spin conversion of $\mathrm{CH}_{3} \mathrm{OH}$ in solid para-hydrogen. Science 2006, 311, 365-368.

(23) Lozada-Garcia, R. R.; Ceponkus, J.; Chevalier, M.; Chin, W.; Mestdagh, J.-M.; Crépin, C. Nuclear spin conversion to probe the methyl rotation effect on hydrogen-bond and vibrational dynamics. Angew. Chem. Int. Ed. 2012, 51, 6947-6950.

(24) Gutierrez-Quintanilla, A.; Chevalier, M.; Ceponkus, J.; Lozada-Garcia, R. R.; Mestdagh, J.-M.; Crepin, C. Large amplitude motions within molecules trapped in solid parahydrogen. Faraday Discuss. 2018, 212, 499-515.

(25) Pauli, W. The connection between spin and statistics. Phys. Rev. 1940, 58, 716-722.

(26) Gutierrez-Quintanilla, A. Molecules and complexes with hydrogen bond: solvation and photoreactivity in cryogenic matrices. Ph.D., Universite Paris-Saclay, 2016.

(27) Miyamoto, Y.; Tsubouchi, M.; Momose, T. Infrared spectroscopy of chloromethyl radical in solid parahydrogen and its nuclear spin conversion. J. Chem. Phys. 2013, 117, 95109517.

(28) Gutierrez-Quintanilla, A.; Chevalier, M.; Platakyte, R.; Ceponkus, J.; Rojas-Lorenzo, G. A.; Crepin, C. 2-Chloromalonaldehyde, a model system of resonance-assisted hydrogen bonding: vibrational investigation. Phys. Chem. Chem. Phys. 2018, 20, 12888-12897.

(29) Strom, A. I.; Fillmore, K. L.; Anderson, D. T. Hydrogen atom catalyzed ortho-to-para conversion in solid molecular hydrogen. Low Temp. Phys. 2019, 45, 676-688.

(30) Fajardo, M. E.; Tam, S. Rapid vapor deposition of millimeters thick optically transparent parahydrogen solids for matrix isolation spectroscopy. J. Chem. Phys. 1998, 108, 42374241.

(31) Tam, S.; Fajardo, M. E. Ortho/para hydrogen converter for rapid deposition matrix isolation spectroscopy. Rev. Sci. Instrum. 1999, 70, 1926-1932.

(32) Fajardo, M. E. Solid parahydrogen thickness revisited. Appl. Spectrosc. 2019, 73, 14031408. 
(33) Silvera, I. F. The solid molecular hydrogens in the condensed phase - Fundamentals and static properties. Rev. Mod. Phys. 1980, 52, 393-452.

(34) El Idrissi, M. I.; Lievin, J.; Herman, M.; Campargue, A.; Graner, G. The vibrational energy pattern in propyne $\left({ }^{12} \mathrm{CH}_{3}{ }^{12} \mathrm{C}_{2} \mathrm{H}\right)$. Chem. Phys. 2001, 265, 273-289.

(35) McIlroy, A.; Nesbitt, D. J. High-resolution, slit jet infrared spectroscopy of hydrocarbons: Quantum state specific mode mixing in $\mathrm{CH}$ stretch-excited propyne. $J$. Chem. Phys. 1989, 91, 104-113.

(36) McIlroy, A.; Nesbitt, D. J.; Kerstel, E. R. T.; Pate, B. H.; Lehmann, K. K.; Scoles, G. Sub-Doppler, infrared laser spectroscopy of the propyne $2 \mathrm{v} 1$ band: Evidence of z-axis Coriolis dominated intramolecular state mixing in the acetylenic $\mathrm{CH}$ stretch overtone. $J$. Chem. Phys. 1994, 100, 2596-2611.

(37) Portnov, A.; Blockstein, L.; Bar, I. Vibrational structure and methyl C-H dynamics in propyne. J. Chem. Phys. 2006, 124, 164301-164301-164308.

(38) Herzberg, G.: Molecular Spectra and Molecular Structure - Infrared and Raman Spectra of Polyatomic Molecules; Krieger Publishing Company: Malabar, Florida, 1988; Vol. Vol. II.

(39) Hollas, J. M.: High Resolution Spectroscopy; 2 ${ }^{\text {nd }}$ ed.; John Wiley \& Sons, Ltd.: New York, New York, 1998.

(40) Szczepanski, J.; Ekern, S.; Vala, M. Spectroscopy and photochemistry of the $\mathrm{C}_{3} \cdot \mathrm{H}_{2} \mathrm{O}$ complex in argon matrices. J. Phys. Chem. 1995, 99, 8002-8012.

(41) Henfrey, N. F.; Thrush, B. A. A high-resolution study of the $v_{7}$ band of propyne. J. Mol. Spectrosc. 1985, 113, 426-450.

(42) Pracna, P.; Muller, H. S. P.; Urban, S.; Horneman, V.-M.; Klee, S. Interactions between vibrational polyads of propyne, $\mathrm{H}_{3} \mathrm{CC} \equiv \mathrm{CH}$ : Rotational and rovibrational spectroscopy of the levels around $1000 \mathrm{~cm}^{-1}$. J. Mol. Spectrosc. 2009, 256, 152-162.

(43) Es-Sebbar, E.; Jolly, A.; Benilan, Y.; Farooq, A. Quantitative mid-infrared spectra of allene and propyne from room to high temperatures. J. Mol. Spectrosc. 2014, 305, 10-16.

(44) Henfrey, N. F.; Thrush, B. A. A high-resolution study of the $2 v_{9}$ band of propyne. J. Mol. Spectrosc. 1987, 121, 139-149.

(45) Xing, X.; Reed, B.; Lau, K.-C.; Baek, S.-J.; Bahng, M.-K.; Ng, C. Y. Assignment of rovibrational transitions of propyne in the region of $2934-2952 \mathrm{~cm}^{-1}$ measured by twocolor IR-vacuum ultraviolet laser photoion-photoelectron methods. J. Chem. Phys. 2007, 127, 044313-1-5.

(46) Go, J.; Cronin, T. J.; Perry, D. S. A free-jet infrared double resonance study of the threshold region of IVR. The $v_{6}, v_{1}+v_{6}$, and $2 v_{1}$ bands of propyne. Chem. Phys. 1993, $175,127-145$.

(47) Zhao, D.; Linnartz, H. The high-resolution infrared spectrum of the $v_{3}+v_{8}$ combination band of jet-cooled propyne. Chem. Phys. Lett. 2014, 595-596, 256-259.

(48) Nauta, K.; Miller, R. E.: In Atomic and Molecular Beams, the State of the Art; Camparque, R., Ed.; Springer-Verlag: Berlin, 2001; pp 775-792. 
(49) Gutierrez-Quintanilla, A.; Briant, M.; Mengesha, E.; Gaveau, M. A.; Mestdagh, J. M.; Soep, B.; Crepin, C.; Poisson, L. A HElium NanoDroplet Isolation (HENDI) investigation of the weak hydrogen bonding in the propyne dimer $\left(\mathrm{CH}_{3} \mathrm{CCH}\right)_{2}$. Phys. Chem. Chem. Phys. 2018, 20, 28658-28666.

(50) Stancik, A. L.; Brauns, E. B. A simple asymmetric lineshape for fitting infrared absorption spectra. Vib. Spectrosc. 2008, 47, 66-69.

(51) Doney, K. D.; Zhao, D.; Linnartz, H. High-resolution infrared spectra of the $v_{1}$ fundamental bands of mono-substituted ${ }^{13} \mathrm{C}$ propyne isotopologues. J. Phys. Chem. A 2018, 122, 582-589.

(52) Nagels, B.; Bakker, P.; Hermans, L. J. F.; Chapovsky, P. L. Nuclear spin conversion in $\mathrm{CH}_{3} \mathrm{~F}$ at elevated temperatures. Phys. Rev. A 1998, 57, 4322-4326.

(53) Nagels, B.; Schuurman, M.; Chapovsky, P. L.; Hermans, L. J. F. Intermolecular versus intramolecular interactions in nuclear spin conversion: Experiments on ${ }^{13} \mathrm{CH}_{3} \mathrm{~F}-\mathrm{O}_{2} . J$. Chem. Phys. 1995, 103, 5161-5163.

(54) Nagels, B.; Schuurman, M.; Chapovsky, P. L.; Hermans, L. J. F. Nuclear spin conversion in molecules: Experiments on ${ }^{13} \mathrm{CH}_{3} \mathrm{~F}$ support a mixing-of-states model. Phys. Rev. A 1996, 54, 2050-2055.

(55) Michaut, X.; Vasserot, A.-M.; Abouaf-Marguin, L. Temperature and time effects on the rovibrational structure of fundamentals of $\mathrm{H}_{2} \mathrm{O}$ trapped in solid argon: hindered rotation and RTC satellite. Vib. Spectrosc. 2004, 34, 83-93.

(56) Turgeon, P.-A.; Vermette, J.; Alexandrowicz, G.; Peperstraete, Y.; Philippe, L.; Bertin, M.; Fillion, J.-H.; Michaut, X.; Ayotte, P. Confinement effects on the nuclear spin isomer conversion of $\mathrm{H}_{2} \mathrm{O}$. J. Phys. Chem. A 2017, 121, 1571-1576.

(57) Henfrey, N. F.; Thrush, B. A. A high-resolution study of the $v_{3}$ and $2 v_{8}^{0}$ bands of propyne. J. Mol. Spectrosc. 1987, 121, 150-166.

(58) Bode, J. H. G.; Smit, W. M. A.; Visser, T.; Verkruijsse, H. D. The absolute infrared intensities of propyne- $\mathrm{d}_{0}$ and propyne- $\mathrm{d}_{3}$. J. Chem. Phys. 1980, 72, 6560-6570.

(59) Doney, K. D.; Zhao, D.; Bouwman, J.; Linnartz, H. The high-resolution infrared spectrum of the $v_{3}+v_{5}$ combination band of jet-cooled propyne. Chem. Phys. Lett. 2017, $684,351-356$.

(60) Duncan, J. L.; McKean, D. C.; Nivellini, G. D. The harmonic force field of methyl acetylene. J. Mol. Struct. 1976, 32, 255-268.

(61) Anttila, R.; Sahlstro, T.; Jaakkone, S. Investigation of some vibration-rotation bands of methyl acetylene in near-infrared. Spectrochimca Acta 1972, A28, 1615-1623.

(62) Villa, M.; Fusina, L.; Nivellini, G.; Didriche, K.; de Ghellinck, X.; Vaernewijck, d. E.; Herman, M. The infrared spectrum of propyne in the range $6200-6700 \mathrm{~cm}^{-1}$. Chem. Phys. 2012, 402, 14-21.

(63) Baylor, L. C.; Weitz, E.; Hofmann, P. Overtone spectroscopy of propyne and propyne-d ${ }_{1}$. J. Chem. Phys. 1989, 90, 615-627. 
(64) Urban, S.; Pracna, P.; Graner, G. Ground state energy levels of propyne: Conventional approach and Pade approximant. J. Mol. Spectrosc. 1995, 169, 185-189. 
Table 1. Peak Positions and Widths (FWHM) in $\mathrm{cm}^{-1}$ of Propyne $\left({ }^{12} \mathrm{C}_{3} \mathrm{H}_{4}\right)$ Trapped in Solid $\mathrm{pH}_{2}$ at $1.7 \mathrm{~K}$.

\begin{tabular}{|c|c|c|c|c|c|c|}
\hline Mode & $\mathrm{NSI}^{\mathrm{a}}$ & $\Delta K_{v^{\prime}}\left(K^{\prime \prime}\right)^{\mathrm{b}}$ & $\operatorname{gas}^{\mathrm{c}}$ & $\mathrm{pH}_{2}(\mathrm{FWHM})$ & Shift & Ref. \\
\hline $2 v_{10} 0^{0}\left(A_{1}\right)$ & $o / p$ & $Q_{2}(0) / Q_{2}(1)$ & 650.352 & $665.44(0.82)$ & 15.09 & 42 \\
\hline$v_{5}\left(A_{1}\right)$ & $o / p$ & $Q_{1}(0) / Q_{1}(1)$ & 930.277 & $929.81(0.13)$ & -0.46 & 42 \\
\hline $2 v_{9}^{0}\left(A_{1}\right)$ & $o / p$ & $Q_{2}(0) / Q_{2}(1)$ & 1254.347 & $1254.47(0.99)$ & 0.12 & 44 \\
\hline$v_{4}\left(A_{1}\right)$ & $o / p$ & $Q_{1}(0) / Q_{1}(1)$ & 1385.579 & $1384.09(1.04)$ & -1.49 & 41 \\
\hline $2 v_{8}^{0}\left(A_{1}\right)$ & $o / p$ & $Q_{2}(0) / Q_{2}(1)$ & 2066.33 & $2058.65(0.50)$ & -7.68 & 57 \\
\hline$v_{3}\left(A_{1}\right)$ & $o / p$ & $Q_{1}(0) / Q_{1}(1)$ & 2137.87 & 2138.34(0.04), m & 0.47 & 57 \\
\hline$\left(v_{3}+2 v_{9}\right)^{+3}\left(A_{1}\right)$ & $o / p$ & $Q_{1,2}(0) / Q_{1,2}(1)$ & 2336.95 & $2310.35(1.25)$ & -26.60 & 34 \\
\hline$v_{7}+v_{8}+v_{9}\left(A_{1}\right)$ & $o / p$ & $Q_{1,1,1}(0) / Q_{1,1,1}(1)$ & 2760 & $2763.71(0.77)$ & -23.40 & 58 \\
\hline $2 v_{4}\left(A_{1}\right) ?$ & $o / p$ & $Q_{2}(0) / Q_{2}(1)$ & 2760 & $2751.99(3.65)$ & -8.01 & 58 \\
\hline $2 v_{7}^{0}\left(A_{1}\right)$ & ortho & $Q_{2}(0)$ & 2879 & $2870.8(1.1), \mathrm{f}$ & -7.8 & 34 \\
\hline $2 v_{7}^{0}\left(A_{1}\right)$ & para & $Q_{2}(1)$ & & $2871.4(0.9), \mathrm{f}$ & & \\
\hline$v_{2}\left(A_{1}\right)$ & $o / p$ & NR & & 2934.4, sh & & \\
\hline$v_{2}\left(A_{1}\right)$ & para & $Q_{1}(1)$ & 2940.944 & $2934.49(0.15), \mathrm{f}$ & -6.46 & 35,45 \\
\hline $\mathrm{v}_{2}\left(A_{1}\right)$ & ortho & $Q_{1}(0)$ & 2941.000 & $2934.60(0.16), \mathrm{f}$ & -6.40 & 35,45 \\
\hline$v_{3}+v_{5}\left(A_{1}\right)$ & $o / p$ & NR & & $3065.7, \mathrm{sh}$ & & \\
\hline$v_{3}+v_{5}\left(A_{1}\right)$ & para & $Q_{1,1}(1)$ & 3070.123 & $3066.01(0.04)$ & -4.11 & 59 \\
\hline$v_{3}+v_{5}\left(A_{1}\right)$ & ortho & $Q_{1,1}(0)$ & 3070.141 & $3066.14(0.30)$ & -4.00 & 59 \\
\hline$v_{1}\left(A_{1}\right)$ & $o / p$ & $Q_{1}(0) / Q_{1}(1)$ & 3335.066 & $3328.52(0.07), \mathrm{m}$ & -6.54 & 35 \\
\hline$v_{3}+2 v_{9}^{0}\left(A_{1}\right)$ & $o / p$ & $Q_{1,2}(0) / Q_{1,2}(1)$ & 3381.15 & $3377.93(1.09)$ & -3.22 & 60 \\
\hline $2 v_{2}\left(A_{1}\right)$ & ortho & $Q_{2}(0)$ & 5781 & $5766.77(1.20), \mathrm{f}$ & -14.23 & 37 \\
\hline $2 v_{2}\left(A_{1}\right)$ & para & $Q_{2}(1)$ & & $5769.10(1.11), \mathrm{f}$ & & \\
\hline$v_{2}+2 v_{7}^{0}\left(A_{1}\right)$ & para & $Q_{1,2}(1)$ & & $5815.14(1.61), \mathrm{f}$ & & \\
\hline$v_{2}+2 v_{7}^{0}\left(A_{1}\right)$ & ortho & $Q_{1,2}(0)$ & 5828 & 5814.72(0.99), f & -13.28 & 37 \\
\hline $2 v_{6}^{0}\left(A_{1}\right)$ & para & $Q_{2}(1)$ & & $5906.14(0.89), \mathrm{f}$ & & \\
\hline $2 v_{6}^{0}\left(A_{1}\right)$ & ortho & $Q_{2}(0)$ & 5917.32 & $5906.40(0.85), \mathrm{f}$ & -10.78 & 61 \\
\hline$v_{1}+v_{2}\left(A_{1}\right)$ & $o / p$ & $Q_{1,1}(0) / Q_{1,1}(1)$ & 6275.841 & $6262.86(0.67)$ & -12.98 & 62 \\
\hline$v_{1}+v_{3}+v_{5}\left(A_{1}\right)$ & $o / p$ & $Q_{1,1,1}(0) / Q_{1,1,1}(1)$ & 6399.463 & $6361.05(0.19)$ & -29.18 & 62 \\
\hline $2 v_{1}\left(A_{1}\right)$ & $o / p$ & $Q_{2}(0) / Q_{2}(1)$ & 6567.878 & 6555.13(0.17), m & -12.75 & 62 \\
\hline$v_{1}+v_{3}+2 v_{9}^{0}\left(A_{1}\right)$ & $o / p$ & $Q_{1,1,2}(0) / Q_{1,1,2}(1)$ & 6660.222 & $6652.00(2.11)$ & -8.22 & 62 \\
\hline$v_{9}(E)$ & $o / p$ & $\tilde{v}_{0} / \mathrm{NR}$ & 638.575 & $633.47(0.76)$ & -5.11 & 42 \\
\hline$v_{8}(E)$ & para & $P_{1}(1)$ & 1030.554 & $1029.76(0.11)$ & -0.79 & 42 \\
\hline$v_{8}(E)$ & $o / p$ & NR & & $1029.93(0.06)$ & & \\
\hline$v_{8}(E)$ & $o / p$ & $\tilde{v}_{0} / \mathrm{NR}$ & 1036.148 & $1031.30(0.17)$ & -4.85 & 42 \\
\hline$v_{8}(E)$ & ortho & $R_{1}(0)$ & 1037.384 & $1034.8, \mathrm{sh}$ & -2.58 & 42 \\
\hline$v_{8}(E)$ & ortho & $R_{1}(0)$ & 1037.384 & $1035.05(0.22)$ & -2.34 & 42 \\
\hline$v_{7}(E)$ & para & $P_{1}(1)$ & 1444.678 & $1443.54(0.45)$ & -1.14 & 41 \\
\hline$v_{7}(E)$ & $o / p$ & $\mathrm{NR}$ & & $1444.93(0.46)$ & & \\
\hline$v_{7}(E)$ & $o / p$ & $\tilde{v}_{0} / \mathrm{NR}$ & 1450.271 & $1446.04(0.39)$ & -4.24 & \\
\hline$v_{7}(E)$ & $o / p$ & NR & & $1451.46(0.61)$ & & \\
\hline$v_{7}(E)$ & ortho & $R_{1}(0)$ & 1459.131 & 1454.67(0.12), sh & -4.46 & 41 \\
\hline$v_{7}(E)$ & ortho & $R_{1}(0)$ & 1459.131 & $1455.12(1.06)$ & -4.01 & 41 \\
\hline$v_{7}(E)$ & para & $R_{1}(1)$ & 1472.926 & $1473.88(4.60)$ & 0.96 & 41 \\
\hline $2 v_{7}^{ \pm 2}(E)$ & para & $P_{2}(1)$ & & $2887.17(0.62)$ & & \\
\hline $2 v_{7}^{ \pm 2}(E)$ & ortho & $R_{2}(0)$ & & $2890.08(0.86)$ & & \\
\hline $2 v_{7}^{ \pm 2}(E)$ & para & $R_{2}(1)$ & & $2898.63(0.44)$ & & \\
\hline$v_{6}(E)$ & para & $P_{1}(2)$ & 2965.400 & $2963.15(1.49)$ & -2.25 & 46 \\
\hline$v_{6}(E)$ & para & $P_{1}(1)$ & 2975.259 & $2971.48(0.60)$ & -3.77 & 46 \\
\hline
\end{tabular}




\begin{tabular}{|c|c|c|c|c|c|}
\hline$v_{6}(E)$ & $o / p$ & NR & & $2972.95(0.50)$ & \\
\hline$v_{6}(E)$ & $o / p$ & $\tilde{v}_{0} / \mathrm{NR}$ & 2980.852 & $2975.96(0.53)$ & -4.90 \\
\hline$v_{6}(E)$ & $o / p$ & NR & & $2978.29(0.24)$ & \\
\hline$v_{6}(E)$ & ortho & $R_{1}(0)$ & 2985.666 & $2979.00(0.46)$ & -6.67 \\
\hline$v_{6}(E)$ & ortho & $R_{1}(0)$ & 2985.666 & $2980.37(0.46)$ & -5.30 \\
\hline$v_{6}(E)$ & para & $R_{1}(1)$ & 2995.479 & $2984.93(1.60)$ & -10.55 \\
\hline$v_{6}(E)$ & para & $R_{1}(1)$ & 2995.479 & $2985.5, \mathrm{sh}$ & -9.98 \\
\hline$v_{6}(E)$ & para & $R_{1}(2)$ & 3005.266 & $2997.19(4.32)$ & -8.07 \\
\hline$v_{3}+v_{8}(E)$ & para & $P_{1,1}(1)$ & 3170.484 & $3165.99(0.13)$ & -4.49 \\
\hline$v_{3}+v_{8}(E)$ & $o / p$ & NR & & $3166.17(0.06)$ & \\
\hline$v_{3}+v_{8}(E)$ & $o / p$ & $\tilde{v}_{0} / \mathrm{NR}$ & 3176.077 & $3167.37(0.16)$ & -8.70 \\
\hline$v_{3}+v_{8}(E)$ & $o / p$ & NR & & $3170.90(0.13)$ & \\
\hline$v_{3}+v_{8}(E)$ & ortho & $R_{1,1}(0)$ & 3177.373 & $3171.31(0.20)$ & -6.07 \\
\hline$v_{3}+v_{8}(E)$ & para & $R_{1,1}(1)$ & 3183.759 & $3175.82(0.31)$ & -7.94 \\
\hline$v_{1}+v_{9}(E)$ & para & $P_{\sim, 1}(1)$ & & $3956.55(50)$ & \\
\hline$v_{1}+v_{9}(E)$ & $o / p$ & $\tilde{v}_{0} / \mathrm{NR}$ & 3947.60 & $3957.97(0.32)$ & 10.37 \\
\hline$v_{1}+v_{9}(E)$ & ortho & $R_{1,1}(0)$ & & $3962.04(0.94)$ & \\
\hline$v_{1}+v_{9}(E)$ & para & $R_{1,1}(1)$ & & $3965.97(0.52)$ & \\
\hline$v_{2}+v_{6}^{ \pm 1}(E)$ & $o / p$ & NR? & & $5780.17(1.49)$ & \\
\hline$v_{2}+v_{6}^{ \pm 1}(E)$ & para & $P_{1,1}(1)$ & & $5784.68(0.18)$ & \\
\hline$v_{2}+v_{6}^{ \pm 1}(E)$ & $o / p$ & $\tilde{v}_{0}$ & 5790 & & \\
\hline$v_{2}+v_{6}^{ \pm 1}(E)$ & ortho & $R_{1,1}(0)$ & & $5789.85(7.48)$ & \\
\hline$v_{6}^{ \pm 1}+2 v_{7}^{0}(E)$ & para & $P_{1,2}(1)$ & & $5835.37(0.07)$ & \\
\hline$v_{6}^{ \pm 1}+2 v_{7}^{0}(E)$ & $o / p$ & $\tilde{v}_{0}$ & 5852 & & \\
\hline$v_{6}^{ \pm 1}+2 v_{7}^{0}(E)$ & ortho & $R_{1,2}(0)$ & & $5841.14(1.05)$ & \\
\hline $2 v_{6}^{ \pm 2}(E)$ & para & $P_{2}(1)$ & & $5938.42(0.75)$ & \\
\hline $2 v_{6}^{ \pm 2}(E)$ & $o / p$ & NR & & $5939.62(0.42)$ & \\
\hline $2 v_{6}^{ \pm 2}(E)$ & $o / p$ & $\tilde{v}_{0} / \mathrm{NR}$ & 5953 & $5941.89(0.28)$ & -11.11 \\
\hline $2 v_{6}^{ \pm 2}(E)$ & $o / p$ & NR & & $5945.38(0.48)$ & \\
\hline $2 v_{6}^{ \pm 2}(E)$ & ortho & $R_{2}(0)$ & 5958 & $5947.05(0.82)$ & -10.95 \\
\hline $2 v_{6}^{ \pm 2}(E)$ & ortho & $R_{2}(0)$ & 5958 & $5948.27(0.37)$ & -9.73 \\
\hline $2 v_{6}^{ \pm 2}(E)$ & para & $R_{2}(1)$ & & $5792.66(0.46)$ & \\
\hline$v_{1}+v_{6}^{ \pm 1}(E)$ & para & $P_{1,1}(1)$ & & $6284.58(0.03)$ & \\
\hline$v_{1}+v_{6}^{ \pm 1}(E)$ & $o / p$ & $\tilde{v}_{0} / \mathrm{NR}$ & 6315.786 & $6286.61(0.15)$ & -29.18 \\
\hline$v_{1}+v_{6}^{ \pm 1}(E)$ & ortho & $R_{1,1}(0)$ & & $6288.82(0.14)$ & \\
\hline
\end{tabular}

${ }^{a}$ Nuclear Spin Isomer: ortho and para spin isomers; $o / p$ indicates unresolved contributions. ${ }^{b} \mathrm{NR}$ stands for non-rotating; see text for details. ${ }^{c}$ Taken from literature values or calculated using literature values and ground state spectroscopic constants of Ref. 64 . $\mathrm{f}=$ value from fit; $\mathrm{m}=$ multiple peaks (principal peak listed), $\mathrm{sh}=$ shoulder. 
Table 2. Parameters Determined from Observed Transition Frequencies and POR.

$\begin{array}{ccccc}\text { Parameter } & v_{6}(E) & v_{7}(E) & v_{3}+v_{8}(E) & v_{6}(E), \text { POR } \\ \tilde{v}_{0} / \mathrm{cm}^{-1} & 2976.59 & 1445.50 & 3170.53 & --- \\ A^{\prime} / \mathrm{cm}^{-1} & 3.62 & 5.77 & 4.13 & --- \\ \zeta_{i} & 0.07250^{\mathrm{a}} & -0.31424^{\mathrm{a}} & 0.4052^{\mathrm{a}} & --- \\ A^{\prime \prime} / \mathrm{cm}^{-1} & 5.10 & 1.96 & 4.54 & 2.22(55) \\ K^{\prime \prime} \text { values } & 0,1 & 0,1 & 0,1 & ---\end{array}$

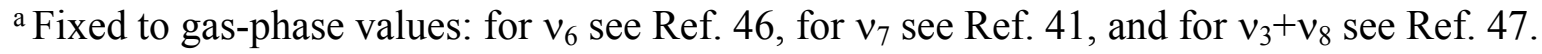




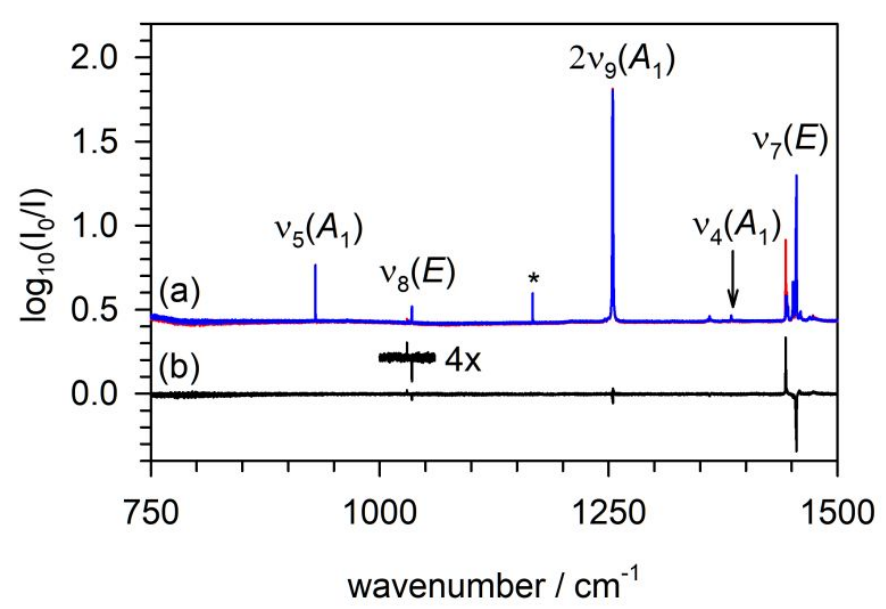

Figure 1. Infrared absorption spectra of propyne trapped in solid $\mathrm{pH}_{2}$ at $1.70(1) \mathrm{K}$ showing propyne absorptions in the $750-1500 \mathrm{~cm}^{-1}$ region. (a) Spectra recorded 2.63 (red) and $491 \mathrm{~min}$ (blue), respectively, after deposition of a 2.5(2) mm thick, 200(39) ppm propyne $/ \mathrm{pH}_{2}$ sample. (b) Difference spectrum $\left(A_{t}-A_{\infty}\right)$ calculated using the two spectra (before - after) displayed in trace (a) showing changes in peak intensities with time. The inset above (b) shows the difference signal multiplied by 4 . The peak marked with * is the $U_{0}(0)$ transition of solid $\mathrm{pH}_{2}$. The $v_{8}(E)$ and $v_{7}(E)$ absorptions display peaks in the difference spectrum consistent with para-to-ortho NSC after deposition. See text for details.

(single column) 


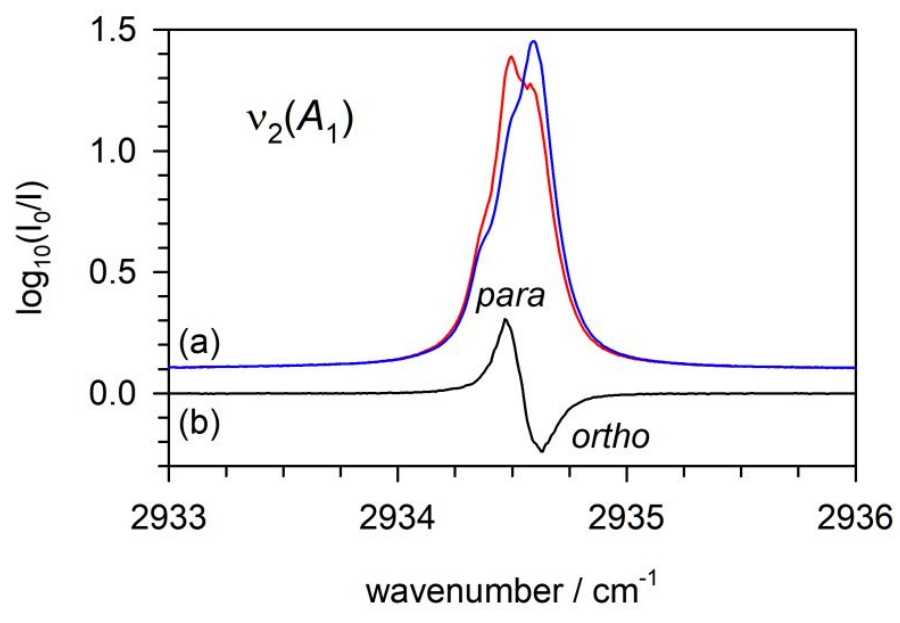

Figure 2. Infrared absorption spectra of propyne in solid $\mathrm{pH}_{2}$ at $1.72(1) \mathrm{K}$ in the region of the $v_{2}\left(A_{1}\right)$ fundamental. (a) Spectra recorded 5.08 (red) and $291.8 \mathrm{~min}$ (blue), respectively, after deposition of the sample shown in Figure 1. (b) Difference spectrum $\left(A_{t}-A_{\infty}\right)$ calculated using the two spectra displayed in trace (a) showing changes in absorption intensity with time after deposition. The positive and negative peaks are labeled para and ortho, respectively, indicating the nuclear spin symmetry in the ground vibrational state associated with each peak. See text for details.

(single column) 


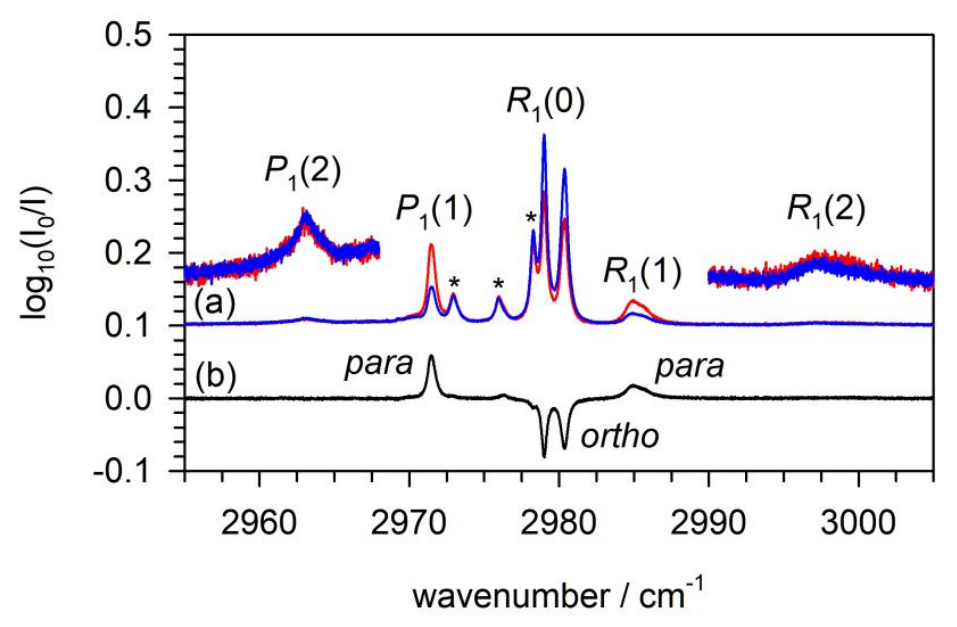

Figure 3. Infrared absorption spectra of propyne in solid $\mathrm{pH}_{2}$ at $1.72(1) \mathrm{K}$ in the region of the $v_{6}(E)$ fundamental. (a) Spectra recorded 5.08 (red) and $291.8 \mathrm{~min}$ (blue), respectively, after deposition of the sample shown in Figure 1. (b) Difference spectrum $\left(A_{t}-A_{\infty}\right)$ calculated using the two spectra displayed in trace (a) showing how the absorption intensity changes with time after deposition. The positive and negative peaks are labeled para and ortho, respectively, indicating the nuclear spin symmetry in the ground vibrational state associated with each peak. Peaks marked with an asterisk do not show up in the difference spectrum. See text for details.

(single column) 


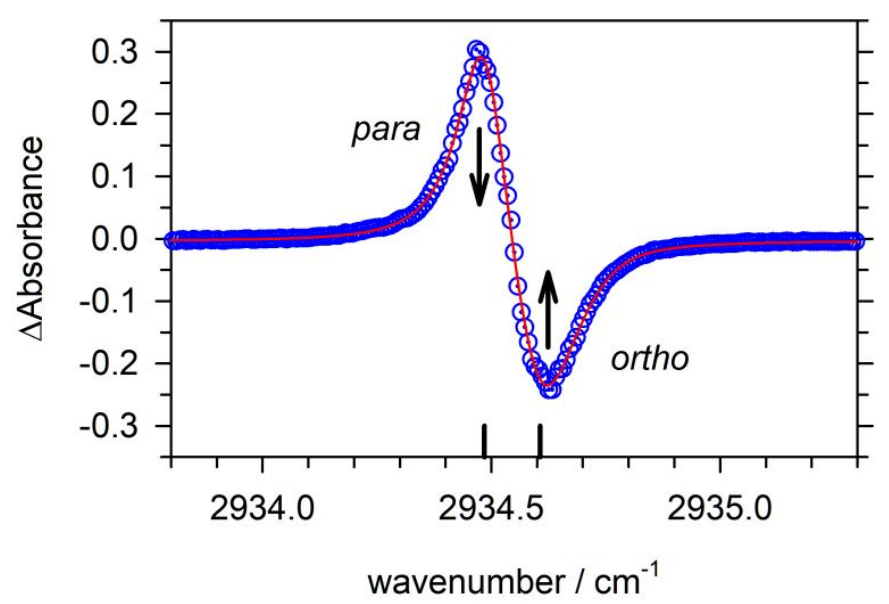

Figure 4. Fits of the $v_{2}$ difference spectrum of propyne in solid $\mathrm{pH}_{2}$ calculated using spectra recorded 5.08 and 291.8 min after deposition of the sample shown in Figure 1. The data are the blue circles and the results of a least squares fit to an analytic function is shown as a red line. The two black lines represent the average peak positions determined from fits to all the spectra for this kinetic run.

(single column) 

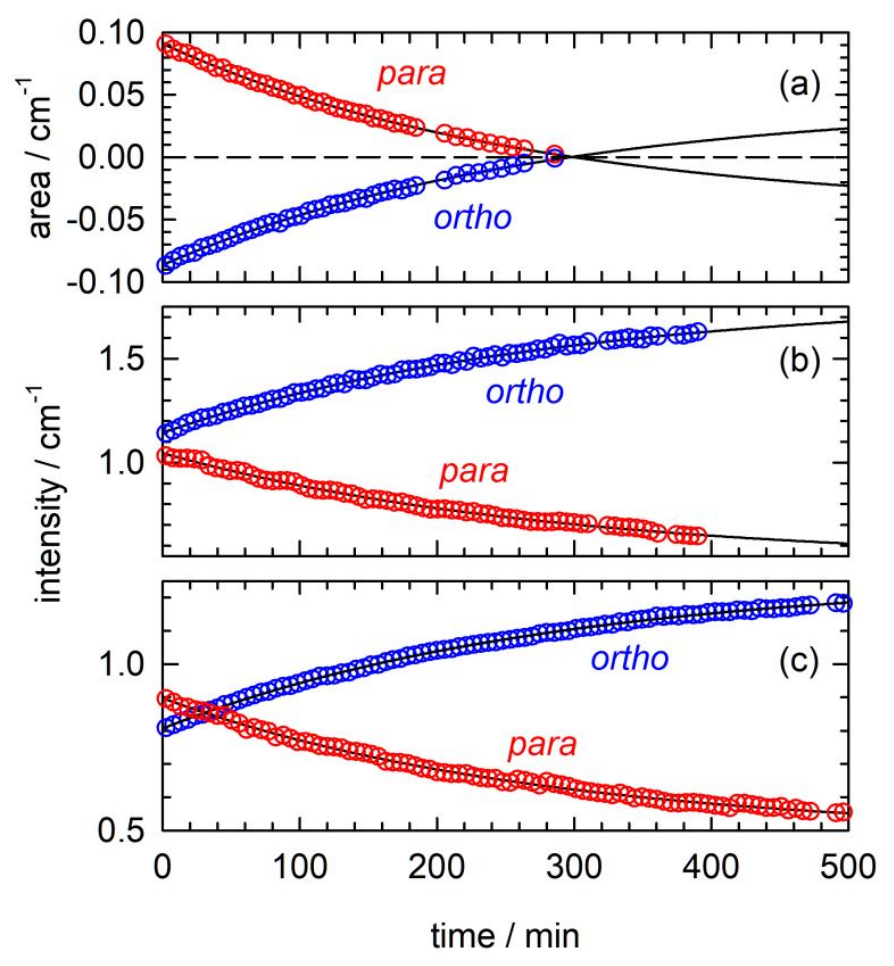

Figure 5. Representative kinetic traces of propyne NSC for propyne isolated in solid $\mathrm{pH}_{2}$ at 1.7 K. (a) The red and blue data points correspond to the areas of the para and ortho peaks in the $v_{2}$ difference spectrum of propyne for the sample shown in Figure 1. Integrated intensities of the ortho and para peaks assigned to (b) $v_{6}$ and (c) $v_{7}$ absorption bands for samples containing 289(16) and 200(39) ppm of propyne, respectively. The red and blue data points in (b) and (c) correspond to the integrated intensities of the para and ortho peaks.

(single column) 


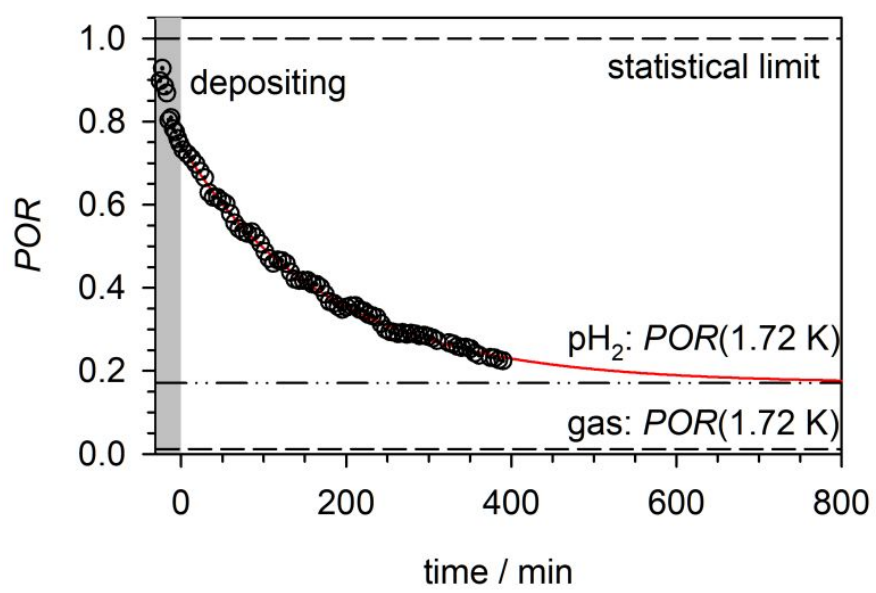

Figure 6. Representative kinetic trace (data shown in Fig. 5b) of the propyne para-to-ortho ratio $(P O R)$ measured as a function of time after deposition for propyne isolated in solid $\mathrm{pH}_{2}$ at $1.7 \mathrm{~K}$.

(single column) 


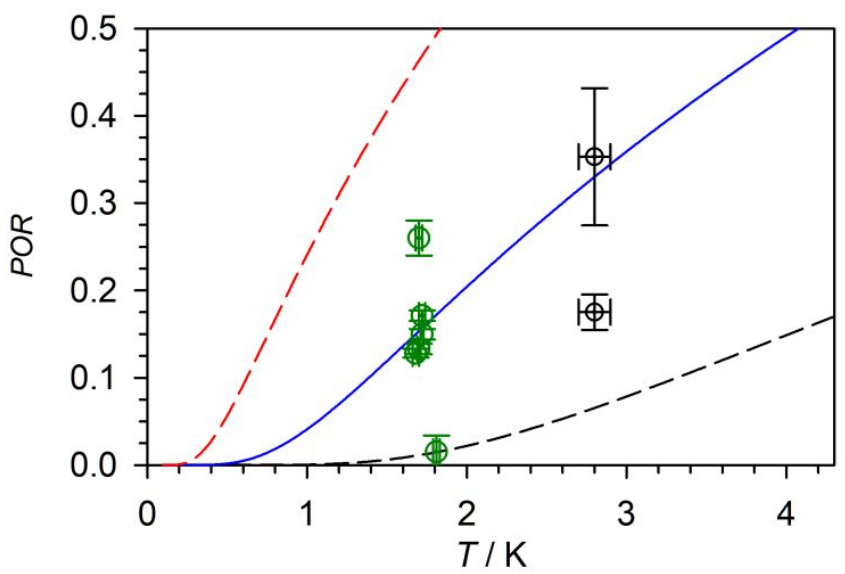

Figure 7. Plot of the equilibrium $P O R$ versus temperature $(\mathrm{K})$ measured in this study. Data from samples grown in Wyoming and Orsay are plotted as green and black circles, respectively. The lines represent theoretical POR curves calculated for single-axis $K$-rotation with effective $A^{\prime \prime}$ constants equal to $1.00 \mathrm{~cm}^{-1}$ (red dashed line), $2.22 \mathrm{~cm}^{-1}$ (blue line), and $5.31 \mathrm{~cm}^{-1}$ (black dashed line). All the measured values lie between the two limiting cases and are best represented by $A^{\prime \prime}=2.22 \mathrm{~cm}^{-1}$. See text and Supporting Information, Tables S6 and S7 for more details. 